\title{
Translation complex profile sequencing to study the in vivo dynamics of mRNA-ribosome interactions during translation initiation, elongation and termination
}

\author{
Nikolay E Shirokikh, Stuart K Archer, Traude H Beilharz, David Powell \& Thomas Preiss Print \\ Nature Protocols 12, 697-731 (2017) doi:10.1038/nprot.2016.189 \\ Published online 02 March 2017
}

\begin{abstract}
Messenger RNA (mRNA) translation is a tightly controlled process that is integral to gene expression. It features intricate and dynamic interactions of the small and large subunits of the ribosome with mRNAs, aided by multiple auxiliary factors during distinct initiation, elongation and termination phases. The recently developed ribosome profiling method can generate transcriptomewide surveys of translation and its regulation. Ribosome profiling records the footprints of fully assembled ribosomes along mRNAs and thus primarily interrogates the elongation phase of translation. Importantly, it does not monitor multiple substeps of initiation and termination that involve complexes between the small ribosomal subunit (SSU) and mRNA. Here we describe a related method, termed 'translation complex profile sequencing' (TCP-seq), that is uniquely capable of recording positions of any type of ribosome-mRNA complex transcriptome-wide. It uses fast covalent fixation of translation complexes in live cells, followed by RNase footprinting of translation intermediates and their separation into complexes involving either the full ribosome or the SSU. The footprints derived from each type of complex are then deep-sequenced separately, generating native distribution profiles during the elongation, as well as the initiation and termination stages of translation. We provide the full TCP-seq protocol for Saccharomyces cerevisiae liquid suspension culture, including a data analysis pipeline. The protocol takes $\sim 3$ weeks to complete by a researcher who is well acquainted with standard molecular biology techniques and who has some experience in ultracentrifugation and the preparation of RNA sequencing (RNA-seq) libraries. Basic Bash and UNIX/Linux command skills are required to use the bioinformatics tools provided.
\end{abstract}

Subject terms: Next-generation sequencing Ribosome RNA RNA sequencing

\section{Introduction}

Mechanisms of gene expression control are central to biology, and there is an increasing appreciation of regulation at the level of RNA ${ }^{1,2}$, particularly through control of mRNA translation ${ }^{3}$. Translation is dysregulated in disease, prominently in cancer ${ }^{4,5,6,7}$. The initiation, elongation and termination phases of translation (Fig. 1) have each been characterized over past decades using the classic tools of biochemistry, genetics and structural biology $8,9,10,11,12,13$. Nevertheless, there are still substantial gaps in our knowledge of translation and its intermediates. This applies in 
particular to the initiation phase in eukaryotes, during which multiple eukaryotic initiation factors facilitate recruitment of the SSU to the mRNA 5' end, scanning of the $5^{\prime}$ untranslated region (UTR), start codon recognition and large ribosomal subunit (LSU) joining to form an elongation-competent ribosome $e^{9,14,15}$.

Figure 1: Overview of the eukaryotic translation cycle of mRNA.

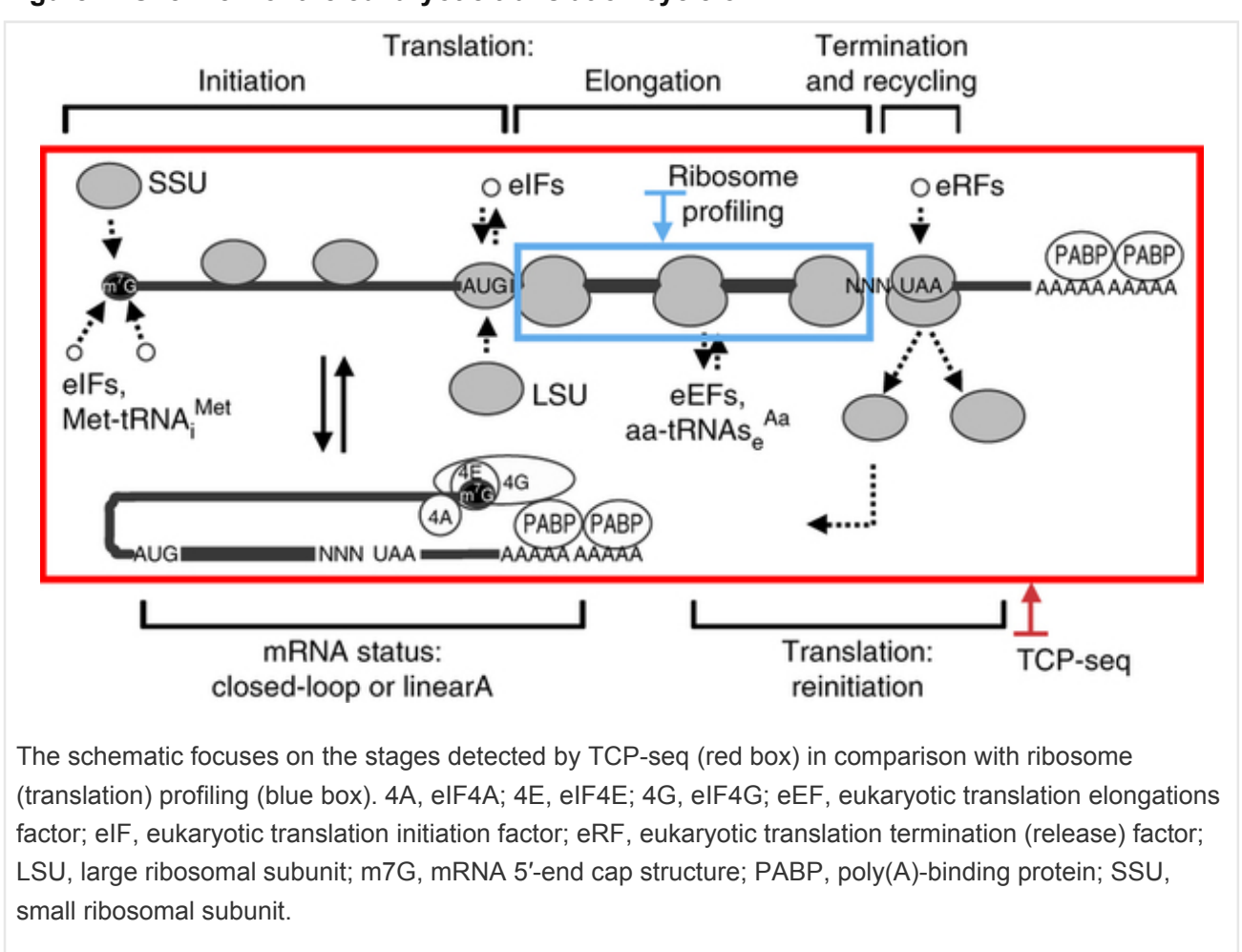

Many regulatory mechanisms are known to affect the capacity of SSUs to reach and recognize start codons, and are thus directly responsible for implementation of gene expression control by setting the level of translation individually for each $\mathrm{mRNA}^{16}$. Classic examples of such control are upstream open reading frames (uORFs) ${ }^{17}$, protein binding elements ${ }^{18}$, structured or excessively short $5^{\prime}$ UTRs $^{19,20,21,22}$ that can negatively affect efficiency of translation, internal ribosome entry sites (IRESes) $^{23,24,25}$, and regions of direct ribosomal attachment ${ }^{26,27}$, as well as strong nucleotide context $^{28,29}$ of initiation sites and downstream secondary structure ${ }^{30}$ that can boost initiation. The recent discoveries of $N^{6}$-methyladenosine sites in mRNA that can recruit elF3 independently of the cap structure ${ }^{31}$, or the unexpected direct cap-binding by elF3 that can bypass the need for the capbinding factor elF4E (ref. 32) suggest an even higher degree of complexity for initiation regulatory mechanisms.

The ribosome profiling approach (Figs. 1 and 2) is thus far the main method of generating footprints of translation transcriptome-wide ${ }^{33,34}$. The approach typically involves arresting translation by brief incubation of cells with an elongation inhibitor, followed by cell lysis, endonucleolytic digestion of mRNA and recovery of full ribosomes in association with protected mRNA footprints, which can then be sequenced. Ribosome profiling has provided several breakthrough discoveries ${ }^{33,} 35,36,37$, elucidating the extensive use of $\mathrm{uORFs}^{38,39,40}$, the occurrence of out-of-frame translation initiation $^{41,42}$ and transcriptome-wide evidence that initiation is generally rate-limiting for translation ${ }^{43}$. Nevertheless, because it detects only fully assembled ('80S') ribosomes, the distribution of translation intermediates involving only the SSU is not recorded (Fig. 1). Even though ribosome profiling can visualize aspects of initiation and termination involving the full ribosome, these are assayed in the presence of specific inhibitors (e.g., cycloheximide, harringtonine), which may affect native distribution of these complexes. Importantly, except for uORFs and alternative start codon usage, mechanisms that regulate initiation, such as steric blocks to SSU scanning 
(mRNA sequence/structure, presence of RNA-binding proteins) and sites of slowed or accelerated SSU motion over 5' UTRs are not visible in ribosome profiling data ${ }^{4}$.

Figure 2: Comparative overview of the TCP-seq and ribosome profiling methods.

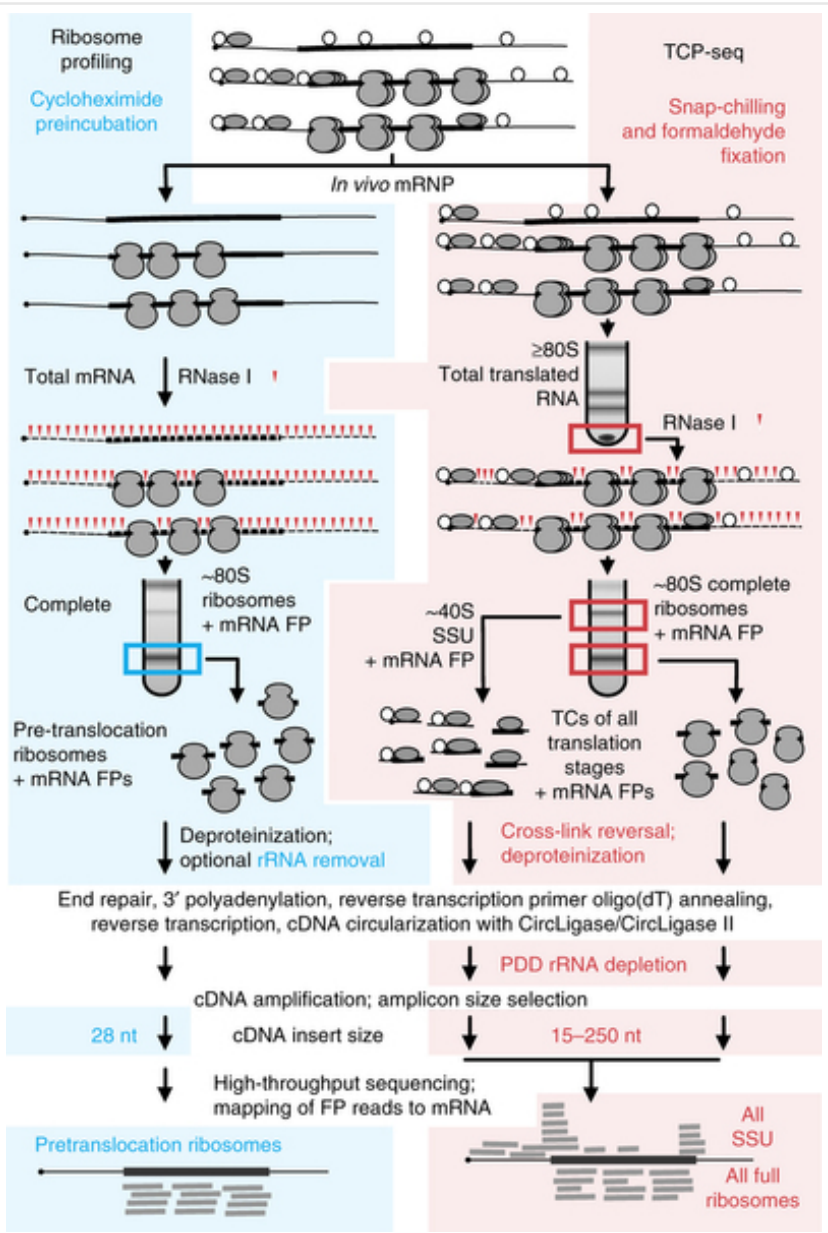

Schematic of ribosome profiling method is depicted on the left, and comparable stages of TCP-seq on the right. Steps with critical differences are highlighted with blue (ribosome profiling) and red (TCP-seq) boxes and explained in the text labels. FP, footprint; mRNP, messenger ribonucleoprotein; PDD, probe-directed degradation.

Here we provide a detailed protocol for the TCP-seq method, as previously published in our transcriptome-wide study of translation in live yeast cells ${ }^{45}$. We developed TCP-seq with the specific intent to profile translation complexes involving SSUs, thus removing limitations for analyses of initiation and post-termination intermediates (Figs. 1 and 2). For example, TCP-seq allows the resolution of intermediates of several substeps of initiation, including the formerly elusive $5^{\prime}$ UTR 'scanning' process and distinct start codon recognition complexes ${ }^{44,45}$. TCP-seq also detects footprints from recycling SSUs located at stop codons ${ }^{45}$. Nevertheless, footprints of full ribosomes are also collected by TCP-seq, and it can thus substitute for ribosome profiling ${ }^{44,46}$. We provide a detailed, step-by-step protocol that was developed with easy replication in mind, discuss limitations and avenues for future development of the method, and outline an overview of a spectrum of possible applications of TCP-seq.

\section{Development and overview of the TCP-seq method}

TCP-seq was inspired by the ribosome profiling approach ${ }^{34}$ (Fig. 2), but it is more specifically built on three long-known biochemical observations. First, ribosomal subunits, complete ribosomes and polyribosomes (polysomes) can be physically separated into different fractions by density gradient ultracentrifugation ${ }^{47,48}$. Isokinetic (or nearly so) sucrose gradients are a traditional tool for 
facilitating separation by sedimentation properties and are widely ysed in analyzing polysomal complexes $^{49,50,51}$. Second, complete ribosomes and SSUs tightly associate with translated $m R N A^{52}$, thus protecting a portion of the mRNA (the ribosomal 'footprint') from nuclease attack ${ }^{53}$. This was observed for elongating ribosomes ${ }^{54,55}$ and for initiating SSUs during aberrant or IRESpromoted initiation ${ }^{56,57,58}$, although protection by scanning SSUs in vivo or in non-modified in vitro translation systems was not reported before. Third, formaldehyde is a reagent of choice for cell fixation and induces covalent cross-links at sites of direct interactions between nucleic acids and proteins $^{59,60,61,62,63}$. Formaldehyde cross-linking is extensively used to study DNA-protein interactions, including in works in which high temporal resolution was achieved ${ }^{64,65}$. Importantly, formaldehyde-induced cross-links are readily reversed by heating of the sample ${ }^{66}$. Thus, it is possible to retrieve DNA fragments cross-linked to specific proteins via affinity purification and to deblock and sequence them, giving sites of the DNA-protein interactions, as is done at a highthroughput scale in chromatin immunoprecipitation sequencing (ChIP-seq) $)^{67,68}$. Direct prompts to the development of TCP-seq were the successful use of fast formaldehyde fixation for other studies on RNA metabolism-e.g., to observe formation of the closed-loop structure of mRNA in live cells ${ }^{69}$, or to 'freeze' several evasive intermediates of ribosome maturation in vivo ${ }^{70}$. Importantly, it allows both full ribosomes and SSUs to be fixed to MRNA; the latter being evident from the presence of 'half-mer' peaks in polysome profiles ${ }^{71}$.

Thus, TCP-seq (Fig. 2) starts with snap-chilling of a yeast suspension culture by adding crushed ice and immediately cross-linking with formaldehyde ${ }^{45}$. Most biochemical reactions are known to slow upon cooling, and so does translation ${ }^{72,73}$. As formaldehyde reactivity with organic amines has the inverse tendency ${ }^{74,75}$, this favors fast cross-linking of complexes to mRNA with minimal distortion of their native distribution. Cell lysates are then prepared by freezing and grinding, and subjected to a first sucrose density gradient ultracentrifugation step. Here, the cross-linked 'translated RNA pool', including polysomes and mRNA associated with one complete ribosome, is concentrated into the pellet, whereas other messenger ribonucleoproteins (mRNPs), as well as free SSUs and LSUs, remain in the supernatant, reducing rRNA contamination of the subsequent sequencing libraries. A small aliquot of the resuspended translated RNA pool is taken for direct high-throughput RNA-seq, creating a reference coverage for all expressed regions of mRNA, whereas the bulk of it is subjected to limited nuclease digestion to remove unprotected portions of mRNA.

Ultracentrifugation through a second sucrose density gradient then results in separate fractions of SSU and full ribosomal complexes, each associated with their respective protected mRNA footprints. Thus, the use of fast covalent cross-linking and the separation of full ribosome and SSU fractions are distinguishing features of TCP-seq, as compared with ribosome profiling ${ }^{46}$.

RNA fragments from the SSU and full ribosome fractions are next deblocked and deproteinized using hot acid-phenol extraction ${ }^{69}$ in preparation for RNA-seq. The requirements for TCP-seq differ from those of other RNA-seq approaches in that during the conversion of the RNA fragments to a deep-sequencing-compatible DNA library, it is important that (i) fragment ends are preserved throughout the procedure, as TCP-seq relies on accurate fragment end mapping to discriminate between translation complex types; (ii) minimal sequence bias is introduced, so that complexes from different regions of mRNA can be compared with each other; and (iii) any size-selection step maintains liberal size limits, as translation complexes from all stages differ in RNA protection length, resulting in a broader range $(\sim 17-75 \mathrm{nt})$ than previously observed in ribosome profiling data ( 20 $-30 \mathrm{nt})$.

TCP-seq libraries are prepared by a reverse transcription/circularization approach that includes repair and polyadenylation of RNA fragment ends; reverse transcription from a primer complementary to the poly(A) tracts split by an internal barrier for the DNA polymerase ('splitadaptor primer', in which the oligo(dT) sequence is separated from the PCR adaptor sequence by, e.g., a triethylene glycol spacer); and circularization and strand-specific amplification of the cDNA. Library preparation is then followed by size selection and sequencing ${ }^{34,76}$ (Fig. 2; Supplementary 
Fig. 1). We used a size selection range from minimal mappable lengths and to $\sim 250 \mathrm{nt}$. The wide range in insert sizes of TCP-seq libraries poses a challenge for efficient, yet specific, rRNA

depletion. Thus, we used the probe-directed degradation (PDD) method, which involves cleavage of perfectly matched (10 bp or more $)^{77}$ dsDNA stretches by duplex-specific nuclease (DSN) at the cDNA stage ${ }^{76,78}$. PDD rRNA depletion probes can be designed with high annealing temperature differentials between target and off-target sites genome-wide for yeast, avoiding the introduction of library bias ${ }^{78}$.

The computational analysis of TCP-seq data also carries different priorities as compared with those of RNA-seq data. Footprint sizes are of prime importance; therefore, read alignments with mRNA sequences must be carefully processed to infer the span of the original fragments. The $3^{\prime}$ polyadenylation step of library generation, although low in bias, also generates its own challenges when read alignments terminate over naturally occurring oligo $(A)$ stretches in the mRNA. We provide the full corresponding software to map and analyze TCP-seq data in a ready-to-use package together with this protocol, publicly available for download at http://bioinformatics.erc.monash.edu/TCPseq/vm/TCPseq.box. Mapping of the original TCP-seq data ${ }^{45}$ is included in the GWIPS-viz browser for genome-wide information on protein synthesis (http://gwips.ucc.ie/) $)^{40}$. We have developed a publicly available website for visualization of the original TCP-seq data available at http://bioapps.erc.monash.edu/TCP/. The website allows browsing through all mapped mRNA; analyzing individual footprint locations and coverage on any mRNA of choice for the SSU, and full ribosome and control libraries; and sorting of mRNAs by different parameters, including annotated $5^{\prime}$ UTR length ${ }^{79}$, number of footprints in different mRNA regions and ratio of $5^{\prime}$ UTR to start codon peak coverage (Fig. 3). Additional metrics and options are available, such as visualization of apparent RNA structure as measured by parallel analysis of RNA structure (PARS) ${ }^{80}$, ambiguous mapping of footprint $3^{\prime}$ ends, filtering of mRNAs by number of reads and visualization of start and stop codons. Future TCP-seq outputs can be added to the visualizations currently available at http://bioapps.erc.monash.edu/TCP/.

Figure 3: Overview of the TCP-seq data browser functions.

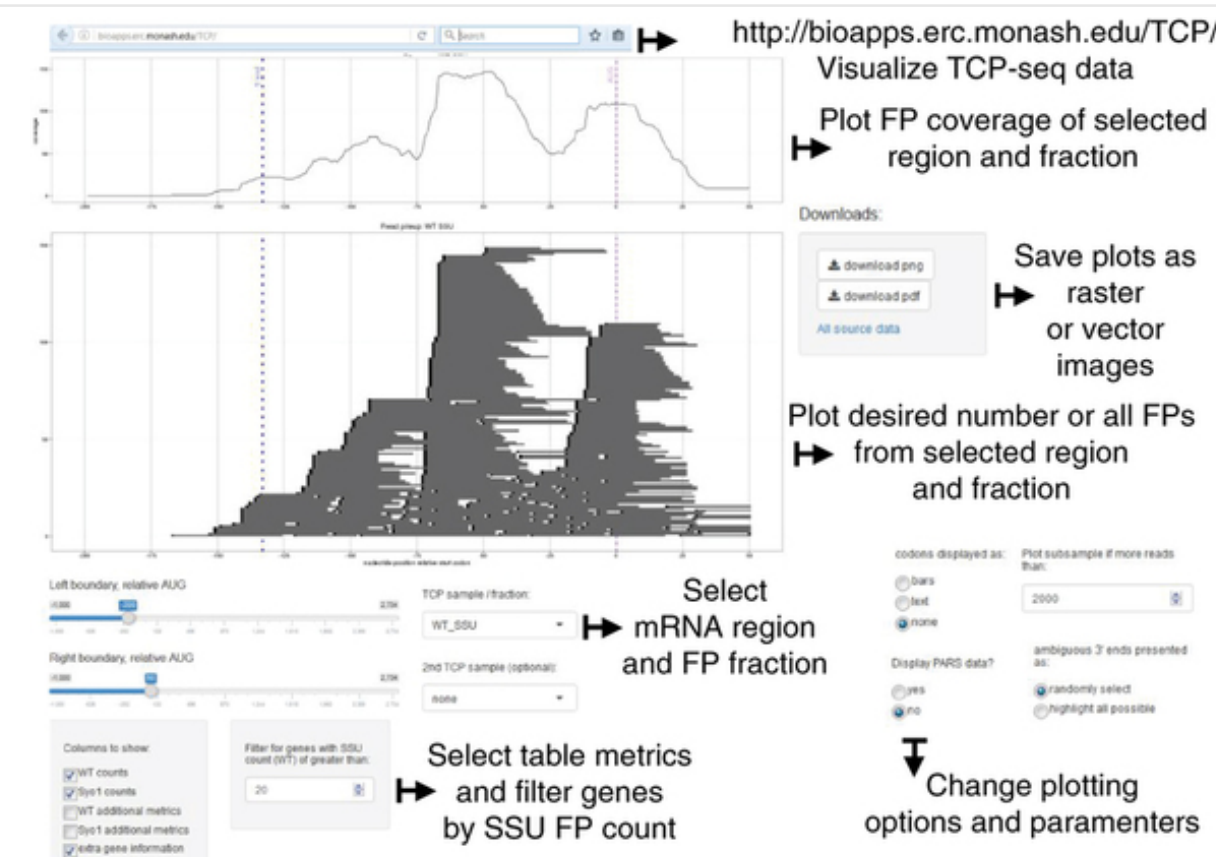

\begin{tabular}{|c|c|c|c|c|c|c|c|c|c|c|c|c|}
\hline \multirow[t]{2}{*}{$\operatorname{sen} x$} & \multicolumn{2}{|c|}{ - notim } & \multicolumn{9}{|c|}{ Search, select and sort transcripts by TCP-seq data 4 - } & \multirow[b]{2}{*}{ Srec_ssun_twom } \\
\hline & $m$ & save ! & oryen: & UTR_. & 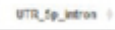 & wryoset: & mys: & wr_sso f & wr_sounsoura & spotfas : & sort_ssa: : & \\
\hline 197 & 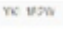 & mast & nisn & vi & tos. & 300 & mas & mes & $10 \mathrm{~m}$ & $m$ & $\infty$ & $m$ \\
\hline แ13 & ravis & พจุ1 & nos & 30 & sit & $m$ & w & $m$ & $6 x$ & $n$ & $m$ & ws \\
\hline 5050 & YPA $45 m$ & $R-01$ & 63 & $2 n$ & tase & 394 & $\mathrm{~s}$ & 196 & 500 & 50 & $3 a t$ & 160 \\
\hline 2010 & vareave & $\mathrm{mst}$ & $2 m$ & 204 & $\operatorname{sen}$ & 1931 & $m$ & 132 & 507 & 70 & $30 x$ & 150 \\
\hline 140 & yeassc & ocont & 45 & & then & 43 & 2 & 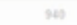 & 46 & 8 & 405 & 100 \\
\hline
\end{tabular}


The browser is publicly available at http://bioapps.erc.monash.edu/TCP/.

We use cookies to improve your experience with our site. Accept and close | More info.

\section{Applications of the TCP-seq method}

The utility of TCP-seq has been demonstrated for liquid suspension culture of yeast cells with a primary focus on aspects of translation initiation, such as the scanning mechanism and dynamics of start codon recognition in vivo ${ }^{45}$. However, TCP-seq should be equally applicable to studies of mRNA-specific translational control ${ }^{81}$. In particular, various environmental conditions known to affect translation initiation, such as nutrient deficiency, alteration of temperature or oxygen supply ${ }^{82}$ ${ }^{83}$, can be tested using TCP-seq to find regulated mRNAs and to identify the responsible mechanisms. In conjunction with numerous function-deficient or temperature-sensitive translation factor mutants available for yeast ${ }^{82}$, and several well-characterized translation initiation inhibitors ${ }^{83}$, ${ }^{84}$, it should be possible to use TCP-seq to further clarify mechanisms of SSU entry and differential initiation factor use across different types of mRNA.

Because TCP-seq generates stable, cross-linked complexes, immunoprecipitation or affinity-tagmediated pulldown in selective buffer conditions can be used in combination with complex isolation by density gradient centrifugation, resembling the ChIP-seq approach. For example, TCP-seq could be used to elucidate points of association and dissociation of individual translation factors along mRNAs, or to detect mRNA-specific translation factor use and occurrence of IRES elements.

TCP-seq should also be applicable to many situations in which ribosome profiling is currently used. Users could simply omit, or delay, the SSU-focused steps and proceed with full ribosome footprint library preparation only, benefiting from additional advantages of TCP-seq, such as close-to-native distribution of the complete ribosomes along mRNAs and resolution of their distinct configurations during the different elongating substeps. Finally, provided that the covalent cross-linking and translation complex extraction stages are optimized accordingly, TCP-seq should also be adaptable to cells or tissues from other eukaryotic or prokaryotic sources. Profiling of in vivo translation complexes in mammalian cells will likely be of high interest, and there should be no principal barrier to applying TCP-seq in this way. However, we anticipate that thorough re-optimization of the crosslinking regimen, as well as subsequent cell lysis, will be required.

\section{Comparison of TCP-seq with other methods}

TCP-seq is currently unique in profiling footprints of intermediates from the complete translation cycle $^{44,45}$. However, its resemblance to ribosome profiling (Fig. 2), and the overlapping goals of gathering data on the distribution of full ribosomes, makes it possible to compare the two methods specifically for this aspect.

Most commonly, ribosome profiling uses translation inhibitors to stall the translation cycle at certain points. Elongation-blocking antibiotics such as cycloheximide or anisomycin are used to block preor post-translocated ribosomes, correspondingly ${ }^{85}$. Combined use of harringtonine and cycloheximide $^{86}$, or lactimidomycin together with puromycin ${ }^{87}$, specifically augments the detection of ribosomes stalled at first codons. Other drugs (e.g., emetine) and nonhydrolyzable GTP analogs have also been used to inhibit the advance of translation ${ }^{86}$. All these treatments lead to accumulation of specific translation intermediates and are thus useful in dissecting substeps of translation. A major recognized problem, however, is that nonuniform inhibitor action prevents the detection of truly native translation complex distribution ${ }^{37,88,89,90,91}$. For instance, early addition of, and incubation with, cycloheximide generates an artificially high abundance of fully assembled ribosomes at start codons (because initiation substeps involving SSUs are not blocked) ${ }^{88,89}$, whereas its late inclusion results in elongation 'run-off' (due to disproportional rates of initiation and elongation upon cell lysis $)^{89,90}$ 


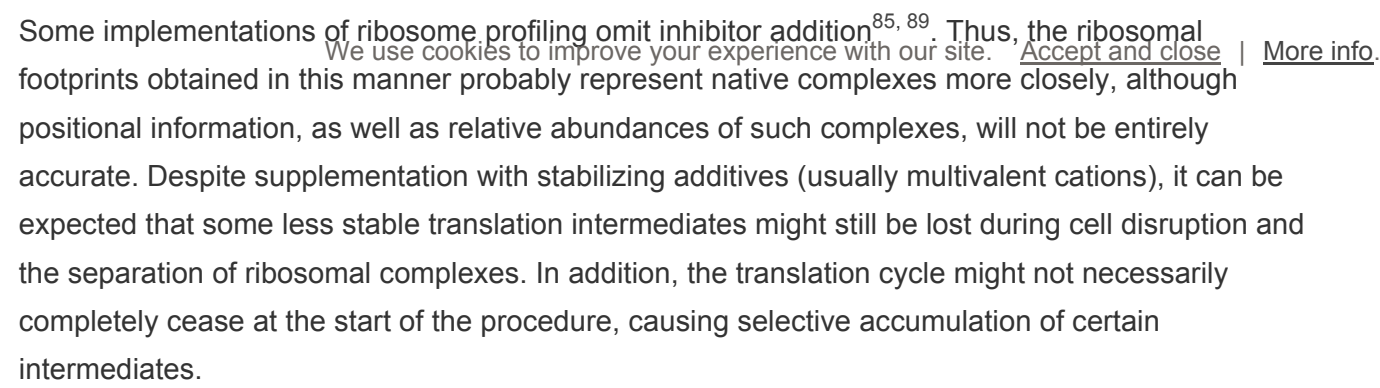

TCP-seq is largely devoid of such problems. It does not force translation complexes into a particular type, allowing observation of the full spectrum of translation intermediates, as they natively occur ${ }^{44}$. For example, both major elongating ribosome configurations (rotated and nonrotated) are seen with TCP-seq (resulting in 21-nt and 31-nt footprints, correspondingly $)^{44,45}$, whereas use of cycloheximide or anisomycin enforces the adoption of one or the other conformation ${ }^{85}$. Formaldehyde reactivity is not known to depend on the translation stage, and as all functional ribosome-mRNA translation complexes develop similar contacts where mRNA is located in the RNA-binding cleft of the SSU ${ }^{92,93,94,95,96}$, they can be expected to cross-link in a similar manner. One key advantage of such stabilization is the ability to obtain footprints from SSUs, which could not be achieved with known inhibitors of translation (e.g., cycloheximide).

Fixation of ribosomal complexes on mRNA can further result in less biased fragment coverage. Slippage of even functional ribosomes was recently described on $m R N A^{97}$. Thus, certain ribosomeprotected fragments of mRNA can still dissociate (slip out) from the nonfixed ribosomes during purification and be underrepresented in the sequencing data, despite the use of translation inhibitors. The prevalence of such effects in ribosome profiling libraries has not yet been systematically investigated; however, a covalent attachment of ribosomal complexes to mRNA as in TCP-seq will guard against such biases.

Although TCP-seq offers the advantages detailed above, it remains a technically more complex method than ribosome profiling. Conditions for formaldehyde cross-linking, cell lysis and the additional physical separation of the complete ribosome and SSU fractions will require specific attention and potentially also reoptimization. Sequencing of an additional footprint library is required. Because of the inclusion of a broader fragment range, more rRNA reads occupy useful sequencing capacity and more sequencing depth is required for the same yield of mRNA footprint counts. Because TCP-seq footprints represent a more complex mixture of different complex subtypes, their deconvolution and quantitative analysis requires additional effort.

\section{Limitations of the method}

A biochemical limitation of the current technique that might easily be eliminated is the separation of the 'translated RNA' by a sedimentation velocity equal to or faster than that of full ribosomes. Although this eliminates unoccupied LSUs and 'empty' nontranslated mRNA/mRNP from the analysis, mRNAs with only single SSUs attached are also probably lost. Valuable information could be gleaned from including these mRNAs, which may represent newly arrived mRNAs, transcripts with a low rate of SSU priming or inactive mRNPS on which initiation was blocked before LSU attachment. The simplest solution (at the expense of meaningful read counts) would be extension of the first sedimentation fraction range to include slower-sedimenting fractions from the $\sim 40 \mathrm{~S}$ region, or processing this fraction in parallel with the SSU and ribosome fractions as currently described. Future advances in rRNA depletion techniques may enhance the feasibility of this modification.

Because of their relatively infrequent occurrence in yeast ${ }^{98}$, we did not attempt to identify mRNA isoforms and quantitatively attribute footprints to them. Several methods have been developed to infer abundances of mRNA isoforms from RNA-seq data ${ }^{99}$, and it seems possible to include a regular RNA-seq control data set and correlate isoform abundances with footprint coverage profiles 


\begin{abstract}
of at least scanning SSUs ${ }^{100,101,102}$. Methods of extracting additional nonribosomal footprint protection sites from ribe output, although we can anticipate better representation of such footprints due to cross-linking.
\end{abstract}

Determining the true $3^{\prime}$ end of footprints whose alignment terminates at one or several adenine residues is complicated because of the $3^{\prime}$ polyadenylation technique used in TCP-seq, as in the original ribosome profiling protocol ${ }^{34}$. However, the ribosome profiling technique was subsequently altered to use ligation of the RNA fragments with preadenylated 3' linkers instead of polyadenylation ${ }^{46}$. Although this approach may incur some fragment end bias resulting from ligation preferences, it provides an unambiguous 3' end identification independent of RNA fragment 3' sequence. We have not yet used such a linker ligation method, but it should work for TCP-seq and could simplify the bioinformatics analysis of the data.

Other limitations are more difficult to circumvent. First, the formaldehyde cross-linking reaction is rapid but not instantaneous. Work with a DNA-binding protein mutated to alter its affinity to, and dissociation rate from, DNA showed strong dependence of cross-linking efficiency on the interaction time between molecules. Complexes that existed for $\sim 2.5 \mathrm{~s}$ were captured at $\sim 50 \%$ efficiency, whereas almost no fixation was observed at interaction times under $2 \mathrm{~s}$ (at room temperature (22 $\left.\left.-25^{\circ} \mathrm{C}\right)\right)^{64}$. Compared with a scanning speed in yeast of $\sim 10 \mathrm{nt} / \mathrm{s}$ and an elongation rate of $\sim 20 \mathrm{nt} / \mathrm{s}$ on average $\left(\text { at } 30^{\circ} \mathrm{C}\right)^{104,105}$, this suggests that some perturbations in the distribution of translation complexes at sites of functional transitions (e.g., start and stop codons) are possible. For example, some SSUs situated closer than 25 nt to start codons at the time of formaldehyde addition might still 'leak' into the start codon recognition state before becoming fixed. There is indeed some tendency for underrepresentation of SSU footprints over short 5' UTRs in the TCP-seq data (Fig. 4). However, there can be other explanations for this observation, and given that chilling substantially increases cross-linking speed while slowing ribosome-related processes, it is a reasonable assumption that any such effects will be minor and not distort TCP-seq profiles in an appreciable way.

Figure 4: Underrepresentation of the SSU footprints in mRNAs with short 5' UTRs. 


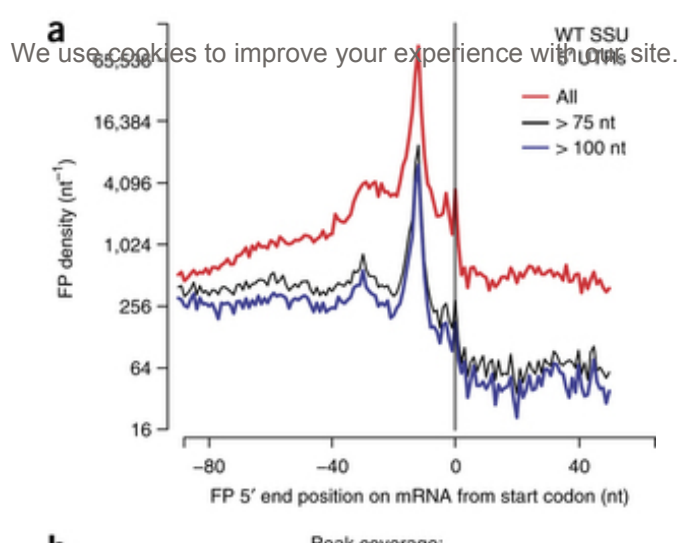

$\underline{\text { Accept and close }} \mid \underline{\text { More info }}$

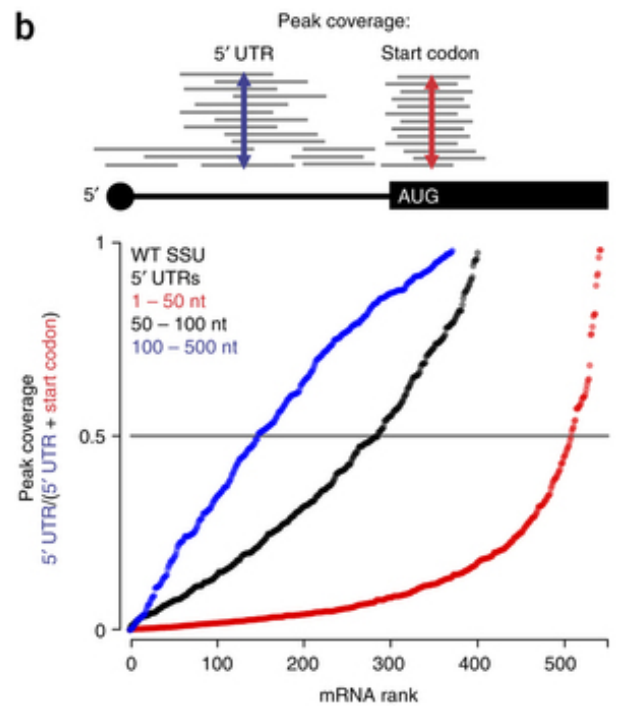

Data taken from the original work on translation dynamics in live yeast cells using TCP-seq ${ }^{45}$. (a) High observed start codon to 5' UTR SSU footprint density is driven by mRNAs with short 5' UTRs. Unambiguous $5^{\prime}$ UTR SSU footprint detection is more difficult on these mRNAs because of short $5^{\prime}$ UTR length. Aggregated profiles of SSU footprint density (SSU library coverage, $y$ axis) are plotted against footprint 5 ' end positions on mRNA (aligned relative to the first ORF nucleotide set at ' 0 ', $x$ axis). (b) Ranking of mRNAs by the ratio of peak SSU footprint coverage in the $5^{\prime}$ UTR to the combined peak SSU footprint coverage in the $5^{\prime}$ UTR and at the start codon. Overrepresentation of mRNAs in the lower ratio band results from mRNAs with short $5^{\prime}$ UTRs (red dots), as compared with mostly equal representation of mRNAs with longer $5^{\prime}$ UTRs (black and blue dots). WT, wild-type.

Second, there is a minimal $5^{\prime}$ UTR length of $\sim 20 \mathrm{nt}$ that is required to reliably discriminate scanning SSUs from the start-codon-associated SSUs. We also cannot exclude the possible existence of very short footprints, especially in the SSU fractions, as unambiguous mapping of fragments $<\sim 17$ nt long in yeast is not feasible. Mapping constraints for genomes of higher eukaryotes will pose even more serious challenges to the mapping of very short footprints.

Third, all ribosome footprinting methods utilize an RNA cleavage step, and thus ribosomes are attacked as well. This compromises ribosome integrity, potentially causing disassembly into subunits and loss of the protected mRNA footprint. Quantifications of such disassembly are not available for ribosome profiling experiments. For the published TCP-seq data ${ }^{45}$, we can estimate a disassembly rate of $\sim 1 \%$ from the molarity of the LSU peak and a roughly equimolar light SSU peak, as compared with the full ribosome peak molarity (Fig. 5). A large proportion of SSU footprints within ORF regions still exhibit codon periodicity ${ }^{45}$, indicating that footprints derived from the small amount of ribosomes that dissociated during purification mostly remain attached to SSUs, probably due to the cross-linking. However, at the level of individual SSU footprints situated within ORFs, this can still create uncertainty as to whether they derive from disassembled complete ribosomes or represent genuine SSU occurrence. Taking into account characteristic footprint 
size/location patterns can help to differentiate genuine ORF-internal scanning SSUs from elongating ribosome-derived SSU footprints. A truly unambiguous identification of the ORF-i initiating SSUs might be possible through tracking translation factor presence in these translation complexes, as suggested above in the 'Applications of the TCP-seq method' section.

Figure 5: Analysis of sucrose gradient UV absorbance profiles.
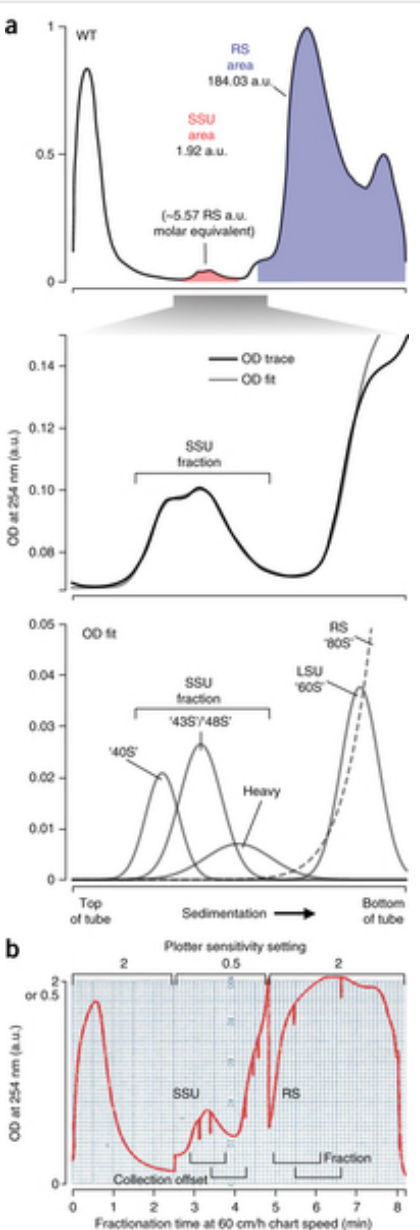

The profiles are taken at $254 \mathrm{~nm}$ after ultracentrifugation to separate SSU and complete ribosome fractions (Steps 54-61) of the total translated RNA fragmented with RNase I. Ultracentrifugation data are taken from the original research on the dynamics of translation in live yeast cells ${ }^{45}$. (a) (Top plot) Estimate of the maximum proportion of the complete ribosomes that dissociate into subunits after RNase treatment during TCP-seq. Note that all SSUs were identified here as a result of such dissociation, whereas our data indicate that less than half of them actually derive from the translating ribosomes. (Middle and bottom plots) Deconvolution of the sucrose gradient sedimentation UV traces in the region of the collected SSU fraction using approximation with Gaussian distribution. (Middle plot) Scaled original UV sedimentation trace (black line) and the result of the fitting of the Gaussian peaks (gray line). (Bottom plot) Gaussian peaks identified from the UV sedimentogram traces. (b) Example of real-time Brandel fractionator plotter output indicating points of sensitivity switching, identified SSU and complete ribosome fractions, and material collection offset taking into account detector-to-fractionator tubing volumes. The figure is a scan of the original plotter output from the Brandel fractionator with superimposed text labels. The plotter marker trace has been isolated, artificially enhanced without changing its geometry or size and superimposed on the original scan for better visibility. OD, optical density; RS, complete ribosomes.

\section{Experimental design}

Figure 6 shows flowchart of a TCP-seq experiment. Because the protocol requires multiple successive enzymatic stages, it is highly recommended that freshly made reagents be used, and ideally proven to be active in advance. For the same reasons, we suggest starting the procedure with $\sim 1 \mathrm{~g}$ of wet cross-linked cell mass to provide for several backup samples, before proceeding with critical or demanding steps. While handling RNA, caution should be taken to use RNase-free 


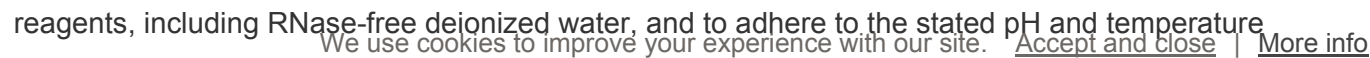
ranges. We recommend first running a control or full ribosome library, for which material is normally plentiful, at least up to the size selection stage, ahead of the complete TCP-seq protocol to verify all enzymatic activities. Precision sucrose gradient fractionation is required to separate the SSU and complete ribosome fractions, and we recommend test-running the equipment before starting the actual procedure. Considerations for several critical stages are outlined below.

Figure 6: Flowchart of the complete TCP-seq method.

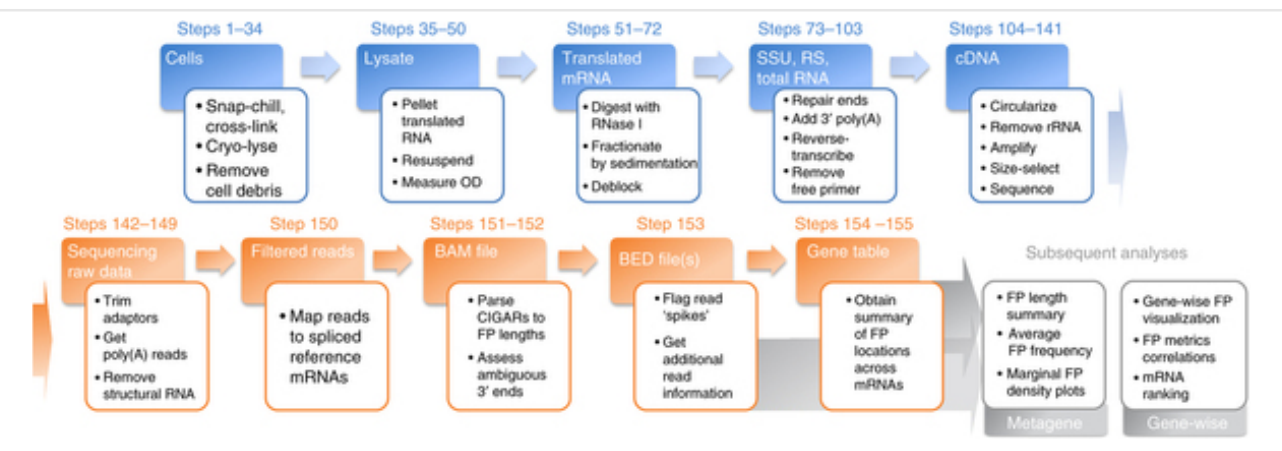

Biochemical steps are shown in blue, and data analysis steps are indicated in amber. Each box includes object and list of applied actions, with the corresponding step numbers of the protocol indicated on top. Several types of subsequent metagene and gene-wise analyses are suggested in the gray boxes for the protocol output data. CIGAR, compact idiosyncratic gapped alignment report.

Optimization of cross-linking. The degree of cross-linking will be affected by the type of cell or tissue being analyzed, and thus we recommend running pilot experiments to test for this. Excessive cross-linking tends to reduce the amount of RNA in the soluble fraction of cytoplasmic extracts, which can be checked by measuring its $O D$ at $260 \mathrm{~nm}\left(\mathrm{OD}_{260}\right)$. A value less than half that of a noncross-linked control may indicate over-cross-linking. Under-cross-linking is more difficult to detect but may be monitored by determining the level of ethylenediamine-tetraacetate (EDTA)-induced SSU/LSU dissociation by sucrose gradient sedimentation (Fig. 7; see 'Separation of the total translated RNA' section).

Figure 7: Fast staining of sucrose density gradients to optimize sedimentation.

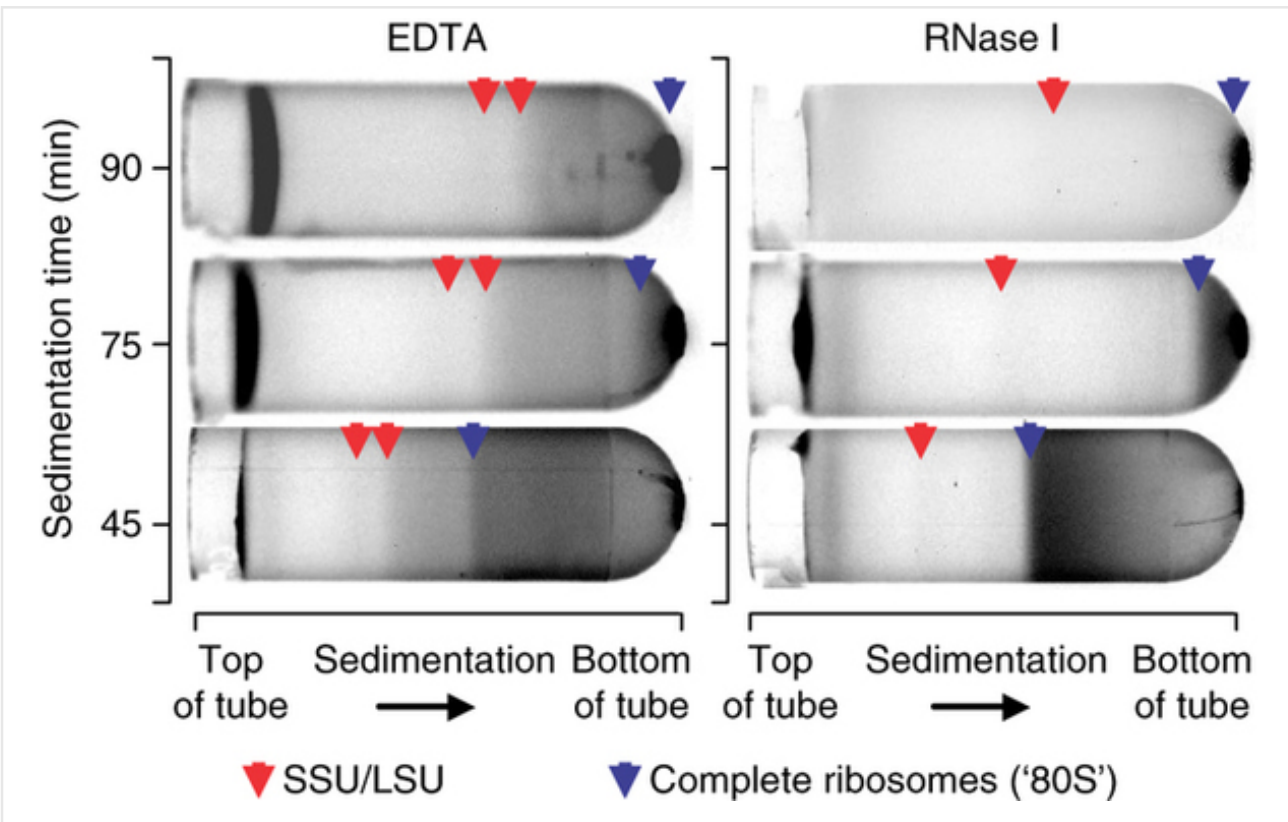

In-tube staining of differently timed sedimentation runs is used to find the optimal sedimentation timing and to estimate cross-linking efficiency and RNase treatment of the total translated RNA. Shown are images of $35-\mathrm{mm}$ density gradients after ultracentrifugation at 52,000 r.p.m. for different times, as indicated on the 
left. Gradients (10-20\% (wt/vol) sucrose; prepared per the first TCP-seq ultracentrifugation step (Steps 26

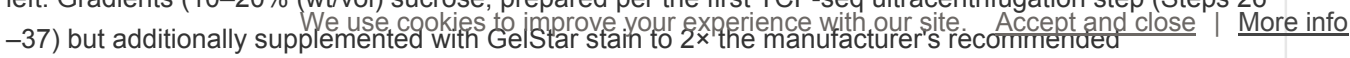
concentration) were loaded with formaldehyde-cross-linked and clarified yeast extracts. Unlike in the regular protocol, extracts (six OD units in $25 \mu \mathrm{l}$ ) were additionally treated at $23^{\circ} \mathrm{C}$ with either EDTA (20 $\mathrm{mM}$ for $15 \mathrm{~min}$, left) or RNase I (60 units for $30 \mathrm{~min}$, right). GelStar stain was added to the resulting mixtures (final concentration $4 \times$ ) to prestain ribosomal complexes. Each mixture was then loaded onto the gradients for centrifugation. Complex positions are indicated by red (SSU/LSU) and blue (full ribosome) arrows. Efficient cross-linking can be ascertained by the presence of EDTA-resistant complexes (left; sedimenting as full ribosomes and faster). Efficient RNase digestion can be devised from the increased intensity of the full ribosome zone and substantial decrease of the faster-sedimenting polysome complexes (right; less material in the pellet as compared to the left). Sedimentation time is chosen to completely pellet full ribosome complexes but not individual subunits.

Inclusion of control libraries. We recommend including at least one control library constructed from the RNA pelleted during the first sedimentation ('total translated RNA'), as it represents the most direct reference for comparing SSU and full ribosome footprint libraries. It is further used to control for fragment cloning bias, evenness of coverage and the presence of possible mapping artifacts. The total translated RNA fraction can be deblocked and deproteinized similarly to the main samples, and fragmented nonspecifically, ideally to the same average fragment length as found in the SSU and full ribosome fractions. Heating in the presence of magnesium ions (such as by using the NEBNext Magnesium RNA Fragmentation Module Kit) or similar methods can be used for the fragmentation. Of note, the ribosome profiling approach typically uses a reference library derived from total cellular RNA to calculate the 'translation efficiency' (TE) parameter. This library is usually poly(A)-selected RNA (poly $(A)^{+}$mRNA or 'total mRNA') ${ }^{34,106}$, but it can also be a size-selected total cellular RNA ${ }^{107}$. In TCP-seq, the ribosome footprint libraries can be related to the total translated RNA library to derive a value somewhat analogous to the TE. However, as the proportion of mRNA molecules recruited to polysomes and the density of ribosomes per mRNA molecule jointly determine translation output, both of these parameters have their limitations, and they are not interchangeable ${ }^{45}$. If users intend to use TCP-seq primarily to profile complete ribosomes, it may be useful to additionally prepare a total cellular RNA ('total mRNA') reference library and calculate conventional TE values to facilitate cross-comparisons with pre-existing ribosome profiling data. However, we do not recommend using solely the data from total cellular RNA ('total mRNA') to supplant the total translated RNA, for the reasons outlined above.

Separation of the total translated RNA. If centrifuge and rotor combinations other than those described here are to be used, then the run time for the initial gradient fractionation (wherein the translated mRNP population is separated from the empty cytoplasmic SSUs) may require optimization. To quickly find a centrifugation running time after which complexes with at least one complete ribosome ('80S') are pelleted but SSUs ('40S') and slower-sedimenting complexes are not, we recommend running a series of differently timed ultracentrifugations in which gradient buffers and samples are supplemented with GelStar (Lonza) stain added at $2 \times$ and $4 \times$ the recommended concentration, respectively. Zones with different sedimentation velocities can then be visualized immediately after sedimentation using a blue-light transilluminator (Fig. 7) to find the correct sedimentation conditions. These tests can also be used to roughly estimate RNase activity by the enhancement of the complete ribosome band relative to the faint SSU and LSU bands after RNase I digestion of the lysate from cross-linked cells. Similarly, cross-linking efficiency can be assessed by stability of the fast-sedimenting polysomal complexes after EDTA pretreatment of the lysate (Fig. 7).

Construction of the cDNA library. Removal of the nonextended excess split-adaptor reverse transcription primer is one of the most technically difficult steps during library construction ${ }^{76}$ as currently used in TCP-seq. The low minimum cDNA insert size makes this particularly challenging, because nonextended, circularized split-adaptor reverse transcription primer will efficiently amplify, the amplification product will be present close to the size-selection target range and it can 
unfavorably affect useful sequencing read counts. That is why we suggest at least a two-stage reverse transcription primer removal (exonuclease I treatment and Agencourt AMPure XP bead purification at high temperature while elongated cDNA is still attached to the polyadenylated RNA fragments ${ }^{76}$ ). It is also important to adhere to the recommended calculations for the ratio of reverse transcription primer to RNA fragments. Higher excess of the primer will result in empty circles with no cDNA; however, lower ratios are also not recommended, as they may bias against less optimally annealing targets. It is possible that more convenient methods of cDNA production can be used for TCP-seq-for instance, the recently developed template-switching ligase-free cDNA construction ${ }^{108}$, 109 (such as TGIRT-seq ${ }^{110,111}$ )—but there is currently less information available about possible fragment sequence bias for these methods.

Depletion of rRNA sequences in cDNA and cDNA size selection. The set of PDD DNA probes targeting rRNA used by us is listed in Supplementary Table 1. This set was composed based on prominent rRNA fragments observed in RNase-I-digested samples of yeast cell lysates. Major rRNA contaminants that remain untargeted can be extracted from either our published ${ }^{45}$ data or the investigator's own data. The set could then be further extended by inclusion of depletion probes that target these contaminants to achieve more efficient rRNA depletion. It should also be possible to restrict the PDD probe set only to SSU rRNA for the SSU fraction treatment. We recommend strictly adhering to the depletion probe set molar ratio to the cDNA library and refraining from modifications of the DSN reaction (especially from increase of incubation time and introduction of other bivalent cations into the mixture), as these may compromise selectivity of the depletion. If a deeper depletion is needed, we recommend using two rounds of PDD, similar to the successive CoThybridization and DSN treatment rounds in use elsewhere ${ }^{112,113,114,115}$. Because total translated RNA or 'total mRNA' (poly $\left.(A)^{+} m R N A\right)$ control libraries are deproteinized and cross-link-reversed before fragmentation, they can be depleted for rRNA using standard methods (Ribo-Zero, RiboMinus and so on) instead of, or in addition to, PDD. It is now established that most of the mappable mRNA footprints are found to be between 17 and $75 \mathrm{nt}$ in length in SSU and complete ribosome TCP-seq libraries ${ }^{45}$. This includes footprints thought to derive from 'queued' double SSUs (peak at $\sim 50 \mathrm{nt}$ ) and stacked double complete ribosomes ( $\sim 60 \mathrm{nt})$. Therefore, size-selection range may be narrowed to a 17- to 75-nt cDNA insert size to further assist in removal of the longer rRNA contaminants.

Sequencing and mapping of the cDNA reads. Any high-throughput sequencing system should be usable for TCP-seq, although the bioinformatics pipeline and the adaptor sequences provided here are tailored to the Illumina HiSeq 2500 system and a read-length of 150 nt. The relative library loading depends on the goal of the experiment; we used an 10:3:4 molar ratio of SSU footprint/complete ribosome footprint/total translated RNA libraries to prioritize the depth of SSU profiles. This can be achieved by amplification of each of these libraries separately with primers containing Illumina barcode sequences unique to each library, mixing them in the required proportion and loading into a single sequencing lane for a multiplexed run. The SSU libraries tend to have a lower proportion of mRNA-mappable reads ( $0.85 \%$ of total raw reads) as compared with the other libraries (full ribosome footprints 1.33\%; total translated RNA 4.80\%). As described here, a sequencing depth of 70 million reads for the SSU library resulted in mRNAs from $\sim 1,100$ yeast genes having at least 20 start-codon-associated footprint alignments.

Because of the high proportion of rRNA- and tRNA-derived inserts in the SSU and ribosome libraries, there is a high a priori likelihood for any read that it is derived from structural RNAs, even if the sequence matches equally well to an mRNA. Therefore, to avoid artifacts arising from mRNA sequences having short local similarity to rRNA sequences, we recommend using a stepwise mapping approach (Fig. 6). Reads are first mapped to structural RNAs, and then only those reads that are completely unmapped in this first round are aligned to mRNAs. The mRNA reference consists of all spliced mRNAs with 1-kbp flanking sequences (forward orientation only and excluding 'dubious' transcript predictions). Postmapping steps include the assessment of footprint $3^{\prime}$ 
ambiguities due to oligo(A) tracts, the classification of footprints according to location within transcripts and the incorporation of other transcript-lev and presence of adjacent transcripts within the 1-kbp flanking regions. Ultimately, the aim is to produce footprint metrics summarized at the transcript level, as well as footprint profiles at the transcript and metagene levels (Figs. 6 and 8 a).

Figure 8: Examples of TCP-seq data analysis and visualization.

a

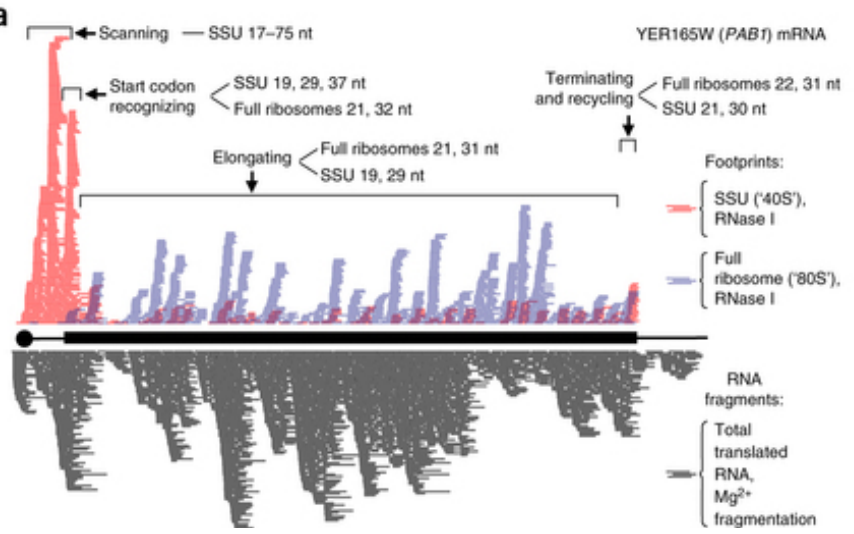

b

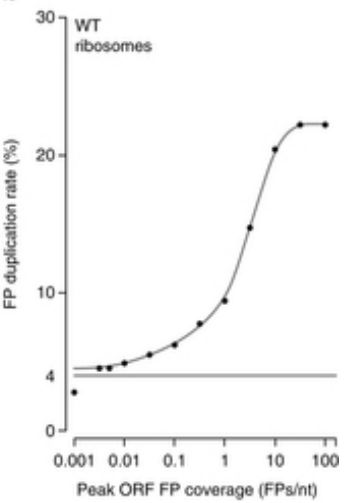

Data taken from the original research on the dynamics of translation in live yeast cells ${ }^{45}$. (a) Experimentally obtained distribution of SSU (red, top) and full ribosome (blue, top) footprints, as well as total translated RNA reads (gray, bottom), along the YER165W (PAB1) mRNA of $S$. cerevisiae resulting from the completion of the TCP-seq protocol as it is described here. Modal lengths of the footprints generated by SSU and complete ribosome translation complexes at each of the different translation stages (initiation, elongation, termination and recycling) are shown above the schematic (derived from transcriptome-wide data). Mapped reads of the footprints and RNA fragments are shown to scale with the mRNA regions (5' UTR, ORF) and are stacked without overlapping. SSU footprints rendered as semitransparent lines and superimposed onto the complete ribosome footprints. (b) Analysis of the WT yeast full ribosome footprint library complexity ${ }^{45}$ by the content of read duplication rate. Read duplication rate was calculated across the ORFs as evenly covered regions (in \%, $y$ axis) for different bins of footprint coverage (per nt of the genome sequence, $x$ axis). As the footprint coverage decreases, the footprint duplication rate approaches $\sim 4 \%$ (indicated by horizontal gray line). WT, wild-type.

The bioinformatics pipeline described here uses preprocessed indices of RNA sequences derived from S. cerevisiae genomic data, gene annotations and other public data sets, which are preinstalled on the downloaded Virtual Machine (genome version numbers can be found in the configuration file). The full set of commands originally used to download the data and generate these indices in the Virtual Machine is given in Step 144. If it is desired to use a different genome version or organism, these commands should be manually rerun from the Virtual Machine after changing their arguments appropriately.

\section{Materials}

\section{REAGENTS}

Critical: Use fresh deionized nuclease-free water to prepare all solutions. Work according to common practices, eliminating risk of contamination of the reagents with nucleases or additional metal ions.

- 2-50 ml of liquid suspension S. cerevisiae BY4741 (ATCC, cat. no. 4040002) culture and/or other desired strain or condition

- Deionized nuclease-free water (such as fresh output from Milli-Q Synthesis, Millipore)

- Adenine hemisulfate (Sigma-Aldrich, cat. no. A9126-25G) 


\section{Caution: Adenine hemisulfate may be toxic. Work under a chemical fume hood
We use cookies to improve your experience with our site. Accept and close
More info or wear an appropriate respirator when handling adenine hemisulfate in dry powder form. Wear personal protective equipment and avoid contact at all stages.}

- Yeast extract (Amresco, cat. no. J850-500G)

Caution: Yeast extract may be allergenic. Work under a chemical fume hood or wear an appropriate respirator when handling dry powder.

- Peptone (Amresco, cat. no. J636-500G)

Caution: Peptone may be allergenic. Work under a chemical fume hood or wear an appropriate respirator when handling dry powder.

- D-Glucose (dextrose; Sigma-Aldrich, cat. no. G7528-250G)

- $\mathrm{NaOH}, 5 \mathrm{M}$ solution, prepared from solid $\mathrm{NaOH}$ and stored at $25^{\circ} \mathrm{C}$ in a tightly closed polypropylene container for no more than 2 weeks (Sigma-Aldrich, cat. no. S8045-500G)

Caution: $\mathrm{NaOH}$ is corrosive and harmful. Wear personal protective equipment (gloves, goggles, laboratory coat) when handling the compound, and dispose of it according to local regulations and considering its corrosive action.

- $\mathrm{pH}$ paper indicator strip (Whatman/Fisher Scientific, cat. no. 09-876-16, or equivalent)

- Crushed ice, $\sim 2 \mathrm{~kg}$

- $\mathrm{HCl}, 6 \mathrm{M}$ solution, $37 \%$ (wt/vol), stored at $25^{\circ} \mathrm{C}$ in a tightly closed polypropylene container for no more than 1 month (Sigma-Aldrich, cat. no. H1758-500ML)

Caution: $\mathrm{HCl}$ is corrosive and harmful. Work under a chemical fume hood when handling concentrated solutions. Wear personal protective equipment when handling the compound (gloves, goggles, laboratory coat), and dispose of according to local regulations and considering its corrosive action.

- Depolymerized 30\% (wt/vol) paraformaldehyde, $100 \mathrm{ml}$, prepared preferably immediately or no more than $1 \mathrm{~d}$ before use (Sigma-Aldrich, cat. no. 441244-1KG, requires: $6 \mathrm{M} \mathrm{HCl}, 5 \mathrm{M} \mathrm{NaOH}$, $\mathrm{NaH}_{2} \mathrm{PO}_{4} \times 2 \mathrm{H}_{2} \mathrm{O}$ powder; see Reagent Setup)

Caution: Paraformaldehyde and formaldehyde are highly toxic, volatile and corrosive. Perform all operations in an appropriate chemical fume hood Wear chemical protective gloves while handling the compound. Do not allow formaldehyde gas to escape into the main laboratory air. Dispose of formaldehyde waste according to your safety regulations and considering its toxicity and volatility.

- $\mathrm{NaH}_{2} \mathrm{PO}_{4}$, powdered (Sigma-Aldrich, cat. no. 71505-250G)

- $\mathrm{KCl}$, solid powder (Sigma-Aldrich, cat. no. P9333-500G)

- $\mathrm{MgCl}_{2}, 1 \mathrm{M}$ solution (Sigma-Aldrich, cat. no. M1028)

- HEPES free acid (Sigma-Aldrich, cat. no. H3375-100G) 
- $\mathrm{KOH}$ solid (Sigma-Aldrich, cat. no. P5958-250G)

We use cookies to improve your experience with our site. Accept and close | More info

Caution: $\mathrm{KOH}$ is corrosive and harmful. Wear personal protective equipment when handling the compound, and dispose of according to local regulations and considering its corrosive action.

- $\mathrm{KOH}, 5 \mathrm{M}$ solution, prepared with solid $\mathrm{KOH}$ stored at $25^{\circ} \mathrm{C}$ in a tightly closed polypropylene container for no more than 2 weeks

Caution: $\mathrm{KOH}$ is corrosive and harmful. Wear personal protective equipment when handling the compound, and dispose of according to local regulations and considering its corrosive action.

- Sodium dodecyl sulfate (SDS; $20 \%$ (wt/vol)) solution (Sigma-Aldrich, cat. no. 05030)

- Ethylenediamine tetraacetate (EDTA) solution, $500 \mathrm{mM}, \mathrm{pH} 8.0$ at $25^{\circ} \mathrm{C}$ (Sigma-Aldrich, cat. no. 03690-100ML)

- Glycine (Sigma-Aldrich, cat. no. G7126-500G)

- DTT, $1 \mathrm{M}$ solution, prepared with solid DL-DTT and stored in a tightly closed polypropylene container at $-20^{\circ} \mathrm{C}$ or below for up to 6 months (Sigma-Aldrich, cat. no. 43815-5G)

Caution: DTT is toxic. Wear personal protective equipment, work under a chemical fume hood or wear an appropriate respirator when handling dry powder.

- Complete EDTA-free Mini Protease Inhibitor (Roche, cat. no. 4693159001)

- RNaseOUT ribonuclease inhibitor (Thermo Fisher Scientific, cat. no. 10777019)

Critical: Do not replace with any RNase inhibitor capable of inhibiting Escherichia coli RNase I.

- Liquid nitrogen

Caution: Protect contacting body parts from low temperature, and work in a wellventilated area to avoid oxygen depletion.

- Tris(hydroxymethyl)aminomethane- $\mathrm{HCl}\left(\right.$ Tris- $\mathrm{HCl}$ ) pH 7.0 at $25{ }^{\circ} \mathrm{C}, 1 \mathrm{M}$ solution (made with Trizma pre-set crystals, Sigma-Aldrich, cat. no. T7193-100G)

- $\mathrm{NH}_{4} \mathrm{Cl}, 1 \mathrm{M}$ solution (made with solid ammonium chloride; Sigma-Aldrich, cat. no. 09718-250G)

- Sucrose (Sigma-Aldrich, cat. no. 84097-250G)

- E. coli ribonuclease I (E. coli RNase I ; Ambion, cat. no. AM2294)

- RNA 6000 Pico Kit (Agilent, cat. no. 067-1513)

- Tris- $\mathrm{HCl}, \mathrm{pH} 7.4$ at $25^{\circ} \mathrm{C}, 1 \mathrm{M}$ solution (made with Trizma Pre-set crystals, Sigma-Aldrich, cat. no. T7693-100G)

- Acidic phenol/chloroform solution (5:1, $\mathrm{pH} \sim 4.5$ at $25^{\circ} \mathrm{C}$; Sigma-Aldrich, cat. no. P1944-100ML)

Caution: Phenol and chloroform are harmful, corrosive and flammable. Avoid contact and always use protective equipment (solvent-resistant gloves, 


\section{protective goggles or face shield). Work in well-ventilated areas or in a
We use cookies toimprove your experience with our site. Accept and close chemical fume hood.}

- NaOAc, $3 \mathrm{M}$ solution, pH 5.2 at $25{ }^{\circ} \mathrm{C}$ (Thermo Fisher Scientific, cat. no. R1181)

- Glycogen solution, 5 mg/ml (Thermo Fisher Scientific, cat. no. AM9510)

- Absolute ethanol (Merck Millipore, cat. no. 1009832511)

Caution: Ethanol is flammable. Avoid generation of aerosol or concentration of vapors.

- Absolute ethanol, 80\% (vol/vol) (Merck Millipore, cat. no. 1009832511), deionized nuclease-free water, $20 \%$ ( $\mathrm{vol} / \mathrm{vol})$ solution

Caution: Ethanol is flammable. Avoid generation of aerosol or concentration of vapors.

- T4 PNK (bacteriophage T4 polynucleotide kinase), $10 \mathrm{U} / \mu \mathrm{l}$ (New England BioLabs, cat. no. M0201S)

Critical: Commonly used 3' phosphatase minus mutants of T4 PNK are not suitable.

- T4 PNK Buffer without ATP (New England BioLabs, cat. no. M0201S)

- $\operatorname{EPAP}(E$. coli poly(A) polymerase), $2 \mathrm{U} / \mu \mathrm{l}$ (Ambion/Thermo Fisher Scientific, cat. no. AM1350M)

- EPAP buffer, 5× (Ambion/Thermo Fisher Scientific, cat. no. AM1350M)

- ATP, 100 mM buffered solution (Ambion/Thermo Fisher Scientific, cat. no. AM1350M)

- Reverse transcription split-adaptor primer, $10 \mathrm{pmol} / \mu \mathrm{l}$ solution (order from IDT, http://www.idtdna.com; dissolve in HE buffer (Reagent Setup); Supplementary Table 1)

- SuperScript III reverse transcriptase, $200 \mathrm{U} / \mu \mathrm{l}$ (Invitrogen/Thermo Fisher Scientific, cat. no. 18080-093)

- DTT, 100 mM solution (Invitrogen/Thermo Fisher Scientific, cat. no. 18080-093)

- First Strand buffer, 5× (Invitrogen/Thermo Fisher Scientific, cat. no. 18080-093)

- dNTP mix, 10 mM each of dATP, dCTP, dGTP and dTTP (Invitrogen/Thermo Fisher Scientific, cat. no. 18427088)

- Exo I (E. coli exonuclease I), 20 U/ $\mu$ l (New England BioLabs, cat. no. M0293S)

- Agencourt AMPure XP reagent (SPRI beads; Beckman Coulter, cat. no. A63880)

- PEG 8000 (poly(ethylene glycol) 8000), 40\% (wt/vol) solution made from solid (Sigma-Aldrich, cat. no. 89510-250G-F)

- Absolute ethanol, 70\% (vol/vol) (Merck Millipore, cat. no. 1009832511) and deionized nucleasefree water, $30 \%$ (vol/vol) solution

Caution: Ethanol is flammable. Avoid generation of aerosol or concentration of vapors.

- $\mathrm{NaCl}, 5 \mathrm{M}$ solution made from solid (Sigma-Aldrich, cat. no. S7653-1KG) 
- RNase A/T (ribonucleases A and T) mix (Thermo Fisher Scientific, cat, no. EN0551)

Critical: Open and work with stock and all subsequent solutions containing the RNase $\mathrm{A} / \mathrm{T}$ mix in an isolated area away from RNA work (i.e., dedicated PCR hood).

- CircLigase II ssDNA (single-stranded DNA) ligase, $100 \mathrm{U} / \mu \mathrm{l}$ (Epicentre, cat. no. CL9025K)

- CircLigase II Buffer, 10× (Epicentre, cat. no. CL9025K)

- $\mathrm{MnCl}_{2}, 50 \mathrm{mM}$ (Epicentre, cat. no. CL9025K)

- Betaine, 5 M (Epicentre, cat. no. CL9025K)

- PDD depletion probes, $1.25 \mathrm{pmol} / \mu \mathrm{l}$ each (order from IDT, http://www.idtdna.com; dissolve in HE buffer (see Reagent Setup); Supplementary Table 1)

- DSN (duplex-specific nuclease) Buffer, 10× (Evrogen, cat. no. EA003)

- DSN, $1 \mathrm{U} / \mu \mathrm{l}$ (Evrogen, cat. no. EA003)

- Phenol/chloroform/isoamyl alcohol with neutral pH (25:24:1, pH 8.0 at $25^{\circ} \mathrm{C}$; Sigma-Aldrich, cat. no. 77617-100ML)

Caution: Phenol and chloroform are harmful, corrosive and flammable. Avoid direct contact; always use protective equipment (solvent-resistant gloves, protective goggles or face shield). Work in well-ventilated areas or in a chemical fume hood.

- Pfx buffer, 10× (Invitrogen/Thermo Fisher Scientific, cat. no. 11708-013)

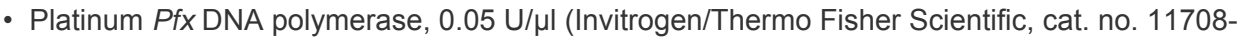
013)

- $\mathrm{MgSO}_{4}, 50 \mathrm{mM}$ (Invitrogen/Thermo Fisher Scientific, cat. no. 11708-013)

- Forward primer, $10 \mu \mathrm{M}$ (order from IDT, http://www.idtdna.com; dissolve in HE buffer (Reagent Setup); Supplementary Table 1)

- Reverse primer, $10 \mu \mathrm{M}$ (order from IDT, http://www.idtdna.com; dissolve in HE buffer (Reagent Setup); Supplementary Table 1)

- ET SSB (extreme thermostable single-stranded DNA-binding protein), $0.5 \mu \mathrm{g} / \mu \mathrm{l}$ (New England BioLabs, cat. no. M2401S)

- GRGreen DNA Loading Buffer, 6× (Excellgen, cat. no. EG-1008)

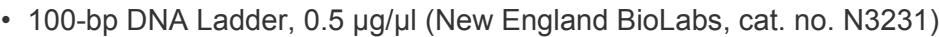

- High-resolution, high-purity agarose (Invitrogen/Thermo Fisher Scientific, cat. no. 16500-500)

- TBE (Tris, borate, EDTA) buffer, $0.5 \times$ (Amresco, cat. no. 0478-2PK; formulate according to the manufacturer's instructions)

- NaOAc, $300 \mathrm{mM}$ solution, pH 8.0 at $25^{\circ} \mathrm{C}$, prepare from $\mathrm{NaOAc}, \mathrm{pH} 5.2$ at $25^{\circ} \mathrm{C}, 3 \mathrm{M}$ solution (Thermo Fisher Scientific, cat. no. R1181)

- High Sensitivity DNA Kit (Agilent, cat. no. 5067-4626)

- TruSeq Small RNA Library Preparation Kit (Illumina, cat. no. RS-200-0012)

- RiboMinus Eukaryote Kit for RNA-Seq (Thermo Fisher Scientific, cat. no. A1083708) 


\section{EQUIPMENT}

We use cookies to improve your experience with our site. Accept and close | More info.

- Water purification system (Millipore, model Milli-Q Synthesis ZMQS6VF01) (or equivalent)

- $0.22-\mu \mathrm{m}$ filter units with bottles (Sigma-Aldrich/Millipore, cat. no. Z660493)

- Schott bottles, 250 ml, 500 ml, 1,000 ml (Sigma-Aldrich/Duran, cat. nos. Z232084, Z232092, Z232106)

- Syringe filters, glass fiber GF/D, 2.7- $\mu$ m pore size (Whatman, cat. no. 6888-2527)

- 60-ml disposable syringe (BD, cat. no. 309653)

- Aluminum foil (Sigma-Aldrich, cat. no. Z691569-1EA)

- Heated magnetic stirrer (Industrial Equipment \& Control, cat. no. 2092-001)

- Magnetic stirring bars, polytetrafluoroethylene (PTFE ) coated (Sigma-Aldrich/Brand, cat. nos. Z329061, Z328952, Z328979, Z329223)

- General-purpose laboratory autoclave (Atherton, model 110A Series Sterilizer or equivalent)

- Ice maker (Hoshizaki, model no. FM-251AFE or similar)

- Chemical fume hood (Laboratory Systems Group, Smoothflow model or equivalent)

- Large (>1.5-liter capacity) glass or polypropylene beaker (Sigma-Aldrich/Corning, cat. no. CLS1000P3L)

- Laboratory and analytical balances (Sartorius, Secura Precision model or Cubis model, cat nos. SECURA313-1CEU, MSE225S-000-DU or equivalent)

- Laboratory vortex (Sigma-Aldrich, Corning LSE model vortex mixer, cat. no. CLS6776 or equivalent)

- $\mathrm{pH}$ meter (Hanna Instruments, model no. $\mathrm{pH} 210$ or equivalent)

- Large (>20-cm) polypropylene stirring rod (Sigma-Aldrich/Nalgene, cat. no. Z275727-1PAK)

- 15-ml conical polypropylene centrifuge tubes (Sigma-Aldrich/Corning, cat. no. CLS430791 or equivalent)

- Parafilm M (Sigma-Aldrich/Brand, cat. no. BR701501-6EA)

- Prechilled $500 \mathrm{ml}$ polypropylene centrifuge bottles with sealed caps (Thermo Fisher Scientific/Nalgene, cat. no. 3141-0500 or equivalent)

- Prechilled fixed-angle centrifuge and rotor for 500-ml tubes (Thermo Fisher Scientific, Sorvall SLA-3000, cat. no. 07149 or equivalent)

- General-purpose large-volume refrigerated centrifuge (Thermo Fisher Scientific, Sorvall Evolution RC Centrifuge, cat. no. 728411 or equivalent)

- Prechilled swinging-bucket rotor and centrifuge for 50-ml conical tubes (Eppendorf Centrifuge $5810 \mathrm{R}$, cat. no. 022628179) (or equivalent)

- Polypropylene or glass formaldehyde waste container ( $\geq 2$-liter capacity; e.g., Sigma-Aldrich, Argos, cat. no. Z688126-1EA)

- Preweighed 50-ml conical polypropylene centrifuge tubes (Sigma-Aldrich, Corning, cat. no. CLS430829-500EA or equivalent) 
- Rack for 15- and 50-Wl centrifuge tubes (Sigma-Aldrich/Scienceware, cat. nos. R8634, R8509 or $\begin{aligned} & \text { Ro } \\ & \text { Me use cookies to improve your experience with our site. Accept and close }\end{aligned}$ equivalent)

- Insulated liquid nitrogen container able to accommodate bead mill canisters and a rack for $50-\mathrm{ml}$ conical tubes (polystyrene foam box $\sim 35 \times 25 \times 20 \mathrm{~cm}(L \times W \times H)$, e.g., VWR, cat. no. 89495-994)

- Pipet aid (Drummond $\mathrm{XP}^{2}$, model no. 4-000-501-I or equivalent)

- Disposable 1-, 2-, 5-, 10-, 25-ml pipettes (Sigma-Aldrich, Corning, cat. nos. CLS4485, CLS4486, CLS4487, CLS4488, CLS4489)

- Set of pipettors $(2,100,200,1,000 \mu$ l; Thermo Fischer Scientific, model Finnpipette F1, cat. nos. $4641010 \mathrm{~N}, 4641060 \mathrm{~N}, 4641080 \mathrm{~N}, 4641100 \mathrm{~N})$

- Pipettor tips (10, 20, 200, 1,000 $\mu$ l; Pathtech, Neptune, cat. no. NEPBT10XLS3/BT10XL; Fisher Scientific, Axygen, cat. nos. 14-222-782/TF20RS, 14-222-778, 14-222-778/TF200RS, 14-222766/TF1000RS)

- General-purpose laboratory -20 and $-80^{\circ} \mathrm{C}$ freezers (SCOPE, model no. SKF1000-DU; Revco, Elite PLUS, model no. ULT2586-6-W46)

- General-purpose laboratory refrigerator (SCOPE, model no. SK650-2-CX: V60BA)

- Laboratory timer (VWR, cat. no. 609-0135)

- Cryo mill with canisters for 1-3 g loads (Retsch, Mixer Mill MM 400)

- 10-ml stainless steel grinding jar (Sigma-Aldrich, cat. no. Z314099-1EA)

- Two 12-mm stainless steel grinding balls (Sigma-Aldrich, cat. no. Z314250-1EA or equivalent)

- Low-binding, polypropylene 1.5-ml microcentrifuge tubes with locking device (Sigma-Aldrich, Eppendorf LoBind, cat. no. Z666548-250EA)

- General-purpose refrigerated micro-volume centrifuge with rotor and adaptors for $1.5-\mathrm{ml}$ and 0.5 ml-microcentrifuge tubes (Thermo Electron Corporation, Heraeus Fresco 17 Centrifuge)

- Scoop-shaped polypropylene or stainless steel microspatula (Sigma-Aldrich, cat. no. Z6482991EA)

- Micro-volume UV-visible spectrophotometer (Thermo Scientific, NanoDrop 1000 Spectrophotometer or equivalent)

- Thin-walled polypropylene $11 \times 35-\mathrm{mm}$ ultracentrifuge tubes (Beckman-Coulter, cat. no. 347357)

- TLS-55 swinging-bucket rotor (Beckman-Coulter, cat. no. 346936)

- Refrigerated ultracentrifuge capable of spinning the TLS-55 rotor (Beckman, Optima L-90K Ultracentrifuge)

- Microcentrifuge tube pestles (Sigma-Aldrich, cat. no. Z359947-100EA)

- NEBNext Magnesium RNA Fragmentation Module (New England BioLabs, cat. no. E6150S)

- Thin-walled polypropylene 89×14 mm ultracentrifuge tubes (Beckman-Coulter, cat. no. 331372)

- 80-mm glass capillary (Sigma-Aldrich/Wheaton Scientific, cat. no. Z114987-200EA or equivalent)

- Silicone tubing, $1 \mathrm{~mm}, \sim 100-\mathrm{mm}$ long (Sigma-Aldrich, cat. no. T1664)

- 5-ml syringe with slip tip (BD, cat. no. 309647) 
- Tube rotator with detachable rotisseries/adaptors accommodating $35 \times 11-\mathrm{mm}$ and $89 \times 14-\mathrm{mm}$. thin-walled polypropylene ultracentrifuge tubes parallel to the axis of rotation (Labnet, Mini LabRoller H5500, Labnet, standard rotisserie model no. H5500, Labnet, additional rotisserie for larger tubes, model no. H5500-15 or equivalent)

- 14-ml round-bottom Falcon polystyrene tubes (Fisher Scientific, Corning, cat. no. 14-959$1 \mathrm{~B} / 352057)$

- SW-41 Ti swinging-bucket rotor (Beckman-Coulter, cat. no. 331362)

- Gradient fractionator with in-line UV absorbance detector (Brandel, Density Gradient Fractionation System, model no. BR-188, based on TELEDYNE ISCO, Density Gradient Fractionator with Foxy R1 fraction collector and UA-6 UV detector, and Brandel, Syringe Pump SYN-202, or equivalent)

- Thermomixer with 1.5- and 0.5-ml microcentrifuge tube blocks (Eppendorf, Thermomixer Comfort, cat. no. 5355 000.011)

- Exchangeable thermoblock for microcentrifuge tubes, $24 \times 1.5-\mathrm{ml}$ (Eppendorf, cat. no. 5360 000.011 or equivalent)

- Exchangeable thermoblock for microcentrifuge tubes, $24 \times 0.5 \mathrm{ml}$ (Eppendorf, cat. no. 5361 000.015 or equivalent)

- 200- $\mu$ l thin-walled nuclease-free polypropylene microcentrifuge tubes (Thermo Fisher Scientific, Ambion, cat. no. AM12225)

- Thermocycler with heated lid (Eppendorf, ep gradient S Mastercycler 5345 or equivalent)

- Magnetic rack for 1.5-ml microcentrifuge tubes (Promega, cat. no. Z5332 or equivalent)

- Dry-heat oven with $\sim 50 \times 30 \times 30-\mathrm{cm}(H \times W \times D)$ internal volume (Bartelt Instruments, model no. EXTRON HI 2001 or equivalent)

- Automated capillary electrophoresis unit (Agilent, 2100 Bioanalyzer, cat. no. G2940CA or equivalent)

- Class II biological safety cabinet (Contamination Control Laboratories, Biological Safety Cabinet Class II or equivalent)

- Low-binding, polypropylene 0.5-ml microcentrifuge tubes with locking device (Sigma-Aldrich, Eppendorf LoBind, cat. no. Z666521)

- General-purpose microwave oven (Panasonic, model no. NNST780W)

- Horizontal gel electrophoresis device (Bio-Rad, Sub-Cell GT Cell, cat. no. 1704401 or equivalent)

- Stabilized power supply for gel electrophoresis (Bio-Rad, PowerPac Universal, cat. no. 1645070 or equivalent)

- Blue-light transilluminator with band-pass or high-band optical filter (Thermo Fisher Scientific/Invitrogen, Safe Imager 2.0, cat. no. G6600 or equivalent)

- 1-ml disposable syringes with sip tip (BD, cat. no. 309659)

- Sharp, clean DNase-free scalpel (Sigma-Aldrich, cat. no. Z692395 or equivalent)

- High-throughput DNA sequencer compatible with Illumina adaptor sequences and barcodes (Illumina, HiSeq 2500) 
- Illustra MicroSpin G-25 columns. (GE Healthcare Life Sciences, cat. no. 27-5325-01)

- Quantum Prep Freeze 'N Squeeze DNA Gel Extraction Spin Columns (Bio-Rad, cat. no. 7326165)

Software requirements

- Linux terminal or Mac running Bash

- Internet connection

- Vagrant (https://www.vagrantup.com/docs/installation/)

- VirtualBox (https://www.virtualbox.org/)

\section{REAGENT SETUP}

Sucrose solution $(60 \%$ (wt/vol))

- To make $500 \mathrm{ml}$, add $300 \mathrm{~g}$ of solid sucrose to a $500-\mathrm{ml} \mathrm{Schott} \mathrm{bottle,} \mathrm{fill} \mathrm{to} 500 \mathrm{ml}$ with deionized water and dissolve the sucrose using a magnetic stirrer and a polytetrafluoroethylene (PTFE)-coated magnetic stirring bar. Using a $0.22-\mu \mathrm{m}$ filter, filter the solution into a sterile bottle and seal the bottle aseptically. Store it at room temperature for up to 6 months.

\section{Paraformaldehyde (30\% (wt/vol))}

- To make $200 \mathrm{ml}$, add $60 \mathrm{~g}$ of paraformaldehyde to a $500-\mathrm{ml}$ Schott bottle and make up to $\sim 175 \mathrm{ml}$ with deionized water. In a chemical fume hood, add $10 \mathrm{ml}$ of $5 \mathrm{M} \mathrm{NaOH}$ to the mixture, cover the bottle with aluminum foil and stir the mixture with heating to $50-60{ }^{\circ} \mathrm{C}$ using a heated magnetic stirrer and a PTFE-coated magnetic stirring bar until fully dissolved. Allow at least $60 \mathrm{~min}$ for this step. Add $4 \mathrm{mmol}(0.63 \mathrm{~g})$ of $\mathrm{NaH}_{2} \mathrm{PO}_{4} \cdot 2 \mathrm{H}_{2} \mathrm{O}$ powder, stir to dissolve and cool to $25^{\circ} \mathrm{C}$. Adjust the $\mathrm{pH}$ to $7.0-8.5$ using slow $(5 \mathrm{ml} / \mathrm{min})$ addition of $6 \mathrm{M} \mathrm{HCl}$ with a pipet aid and a disposable 2-ml pipette, and make up the volume to $200 \mathrm{ml}$ using deionized water. Using a $60-\mathrm{ml}$ syringe and a $2.7-\mu \mathrm{m}$ glass fiber filter, filter the mixture into a clean 250-ml Schott bottle. Replace the filter during filtration if back-pressure becomes excessive. Close the bottle tightly before removing it from the fume hood. Store it at room temperature for up to $2 \mathrm{~d}$.

Caution: Paraformaldehyde is toxic and volatile. $\mathrm{NaOH}$ and $\mathrm{HCl}$ are corrosive. Perform all steps in a chemical fume hood and wear personal protective equipment (goggles, gloves and laboratory coat). Dispose of paraformaldehyde in accordance with local regulations.

Sterile glucose solution $(20 \%$ (wt/vol))

- To make $250 \mathrm{ml}$, add $50 \mathrm{~g}$ of anhydrous glucose to a 250-ml Schott bottle, and make up the volume to $250 \mathrm{ml}$ with deionized, nuclease-free water. Dissolve the glucose using a magnetic stirrer and PTFE-coated magnetic stirring bar. Using a $0.22-\mu \mathrm{m}$ filter, filter the solution into a sterile bottle and seal the bottle aseptically. Store it at room temperature for up to 6 months.

Yeast extract, peptone, adenine, dextrose medium (1x)

- To make 1 liter of yeast extract, peptone, adenine and dextrose (YPAD) medium, combine 10 $\mathrm{g}$ of yeast extract, $20 \mathrm{~g}$ of peptone and $0.4 \mathrm{~g}$ of adenine hemisulfate in a graduated beaker, and make up the volume to $800 \mathrm{ml}$ with deionized water. Dissolve using a magnetic stirrer and a PTFE-coated magnetic stirring bar. Transfer the mixture to a 1-liter or two 500-ml Schott bottles with caps and autoclave for $30 \mathrm{~min}$ at $121^{\circ} \mathrm{C}$. Aseptically (using a biological safety cabinet or similar) adjust the $\mathrm{pH}$ to $7.0-7.5$ (as checked by $\mathrm{pH}$ paper indicator strip) 
with $5 \mathrm{M} \mathrm{NaOH}$ (or $6 \mathrm{M} \mathrm{HCl}$ ) add $100 \mathrm{ml}$ of sterile $20 \%$ (wt/yol) glucose solution and make up to 1 liter with sterile deionized water. Store the solution at $4{ }^{\circ} \mathrm{C}$ for up to 6 months.

Glycine (2.5 M and $250 \mathrm{mM}$ ) solution

- To make $250 \mathrm{ml}$ of $2.5 \mathrm{M}$ glycine solution, add $46.9 \mathrm{~g}$ of glycine to a $250-\mathrm{ml}$ Schott bottle, and make up the volume to $250 \mathrm{ml}$ with deionized, nuclease-free water. Dissolve the glycine using a magnetic stirrer, and heat the solution to $40-50^{\circ} \mathrm{C}$ using a dry-heat oven (or water bath) if necessary. Using a $0.22-\mu \mathrm{m}$ filter, filter the solution into a sterile bottle and seal the bottle aseptically. Store it at room temperature for up to 6 months. Dilute 10 -fold with deionized nuclease-free water to obtain a $250 \mathrm{mM}$ glycine solution.

HEPES-KOH, pH 7.6 at $25^{\circ} \mathrm{C}(1 \mathrm{M})$

- To make $250 \mathrm{ml}$, add $59.6 \mathrm{~g}$ of HEPES free acid to a 250-ml glass beaker, and make up the volume to $200 \mathrm{ml}$ with deionized, nuclease-free water. Dissolve the HEPES free acid using a magnetic stirrer and a PTFE-coated magnetic stirring bar, and adjust the temperature of the mixture to $25^{\circ} \mathrm{C}$ with the stirrer if necessary. Use a pH meter according to the manufacturer's instructions and monitor the $\mathrm{pH}$ continuously while stirring and keeping the temperature stable. Sequentially add solid $\mathrm{KOH}$ pellets, giving enough time for $\mathrm{pH}$ readings to stabilize, until a $\mathrm{pH}$ of $\sim 7.5$ is reached. Using a 1-ml pipettor, adjust the $\mathrm{pH}$ to 7.6 with $5 \mathrm{M} \mathrm{KOH}$ solution, and make up the volume to $250 \mathrm{ml}$ with deionized, nuclease-free water. Using a $0.22-\mu \mathrm{m}$ filter, filter the mixture into a sterile bottle, and seal the bottle aseptically. Store it at 4 ${ }^{\circ} \mathrm{C}$, away from light, for up to 6 months.

HE buffer (1x)

- To make $10 \mathrm{ml}$ of HE buffer, combine $100 \mu \mathrm{l}$ of $1 \mathrm{M} \mathrm{HEPES}-\mathrm{KOH}, \mathrm{pH} 7.6$ at $25^{\circ} \mathrm{C}$, and $2 \mu \mathrm{l}$ of $500 \mathrm{mM}$ EDTA, $\mathrm{pH} 8.0$ at $25^{\circ} \mathrm{C}$, in a new $15-\mathrm{ml}$ conical polypropylene centrifuge tube and make up the volume to $10 \mathrm{ml}$ with deionized nuclease-free water. Store the solution away from light at $4{ }^{\circ} \mathrm{C}$ for up to 1 week or freeze it at $-20^{\circ} \mathrm{C}$ for up to 6 months.

Stop solution $(100 \%$ (vol/vol))

- To make $10 \mathrm{ml}$ of stop solution, combine $5 \mathrm{ml}$ of $20 \%$ (wt/vol) SDS solution and $2 \mathrm{ml}$ of 500 mM EDTA, pH 8.0 at $25^{\circ} \mathrm{C}$, in a new $15-\mathrm{ml}$ conical polypropylene centrifuge tube and make up the volume to $10 \mathrm{ml}$ with deionized nuclease-free water. Store the solution at room temperature for up to 6 months.

Buffer A (10x stock)

- To make $100 \mathrm{ml}$ of $10 \times$ stock, mix $25 \mathrm{ml}$ of $1 \mathrm{M} \mathrm{HEPES}-\mathrm{KOH}, \mathrm{pH} 7.6$ (at $25^{\circ} \mathrm{C}$ ), $7.46 \mathrm{~g}(0.1$ $\mathrm{mol}$ ) of $\mathrm{KCl}$ and $2 \mathrm{ml}$ of $1 \mathrm{M} \mathrm{MgCl}_{2}$ in a clean $250-\mathrm{ml}$ Schott bottle and make up the volume to $100 \mathrm{ml}$ with deionized nuclease-free water. Stir the mixture with a magnetic stirrer and a PTFE-coated magnetic stirring bar until dissolved. Store the buffer at $4{ }^{\circ} \mathrm{C}$ for up to 2 months. To make $1 \times$ Buffer $\mathrm{A}$, dilute $10 \times$ stock as appropriate with deionized nuclease-free water.

Buffer A-1 (1x)

- To make $100 \mathrm{ml}$ of $1 \times$ Buffer A-1, mix $80 \mathrm{ml}$ of deionized nuclease-free water, $10 \mathrm{ml}$ of $10 \times$ stock of Buffer $A$ and $10 \mathrm{ml}$ of $250 \mathrm{mM}$ glycine in a clean 250-ml Schott bottle. Store the buffer at $4{ }^{\circ} \mathrm{C}$ for up to 1 week or at $-20^{\circ} \mathrm{C}$ for up to 2 months.

Buffer A-2 (1x)

- Mix $1 \mathrm{ml}$ of $10 \times$ stock of buffer A, $5 \mu$ l of 1 M DTT ( $0.5 \mathrm{mM}$ final concentration) and one tablet of Complete EDTA-free Mini Protease Inhibitor (Roche) in a new 15-ml conical centrifuge tube and make up the volume to $10 \mathrm{ml}$ with deionized nuclease-free water. Mix by vortexing until the tablet is dissolved, dispense the solution in 1-ml aliquots, freeze them and store them at $-20^{\circ} \mathrm{C}$ for up to 2 months.

Buffer A-3 (1x) 
- Mix $1 \mathrm{ml}$ of $10 \times$ stock of buffer A and $5 \mu$ l of $1 \mathrm{M}$ DTT $(0.5 \mathrm{mM}$ final concentration $)$ in a ney We use cookies to improve your experience with our site. $15-\mathrm{ml}$ conical centrifuge tube and make up the volume to $10 \mathrm{ml}$ with deionized nuclease-free water. Mix the buffer, dispense it in 1-ml aliquots, freeze them and store them at $-20^{\circ} \mathrm{C}$ for up to 2 months. Before use, thaw and add RNase OUT (Life Technologies) to a $1 \times$ recommended concentration (at least, $1 \mathrm{U} / \mu \mathrm{l}$ ).

Buffer B (2x)

- To prepare $500 \mathrm{ml}$ of $2 \times$ buffer B stock, mix $50 \mathrm{ml}$ of $1 \mathrm{M}$ Tris- $\mathrm{HCl}, \mathrm{pH} 7.0,50 \mathrm{ml}$ of $1 \mathrm{M}$ $\mathrm{NH}_{4} \mathrm{Cl}$ and $4 \mathrm{ml}$ of $1 \mathrm{M} \mathrm{MgCl}_{2}$ in a clean 500 -ml Schott bottle and make up the volume to 500 $\mathrm{ml}$ with deionized nuclease-free water. Store the buffer at $4{ }^{\circ} \mathrm{C}$ for up to 1 week or at $-20{ }^{\circ} \mathrm{C}$ for up to 2 months.

Sucrose gradients

- Sucrose should be dissolved in buffer B as detailed in Box 1 to produce 7.5\%, 10\%, 20\%, $23 \%$ and $30 \%(\mathrm{wt} / \mathrm{vol})$ gradients.

\section{Box 1: Preparation of sucrose density gradients • TIMING $\sim 4 \mathrm{~h}$}

Full box

PN binding buffer (1x)

- To make $10 \mathrm{ml}$, combine $5 \mathrm{ml}$ of $40 \%$ (wt/vol) PEG-8000 solution and $5 \mathrm{ml}$ of $5 \mathrm{M} \mathrm{NaCl}$ solution in a new $15-\mathrm{ml}$ conical polypropylene centrifuge tube. Store the buffer at room temperature for up to 6 months.

PN washing buffer (1x)

- To make $12 \mathrm{ml}$ of PN washing buffer, combine $8 \mathrm{ml}$ of $1 \times \mathrm{PN}$ binding buffer and $4 \mathrm{ml}$ of $1 \times$ $\mathrm{HE}$ buffer in a new $15-\mathrm{ml}$ conical polypropylene centrifuge tube. Store the buffer at $4{ }^{\circ} \mathrm{C}$ for up to 6 months.

\section{EQUIPMENT SETUP}

\section{Gradient Fractionator}

- Set up the fractionator in a $4{ }^{\circ} \mathrm{C}$ cabinet ('cold room') according to the manufacturer's recommendations. Equip the UV absorbance unit with a 254-nm band-pass wavelength filter. Use a thin ( 1-mm internal diameter or less) PTFE capillary (supplied with the fractionator) to connect the UV absorbance detection unit and the fraction collector. Ensure that all tubing and the syringe pump are clean and nuclease-free. Prefill the system with $60 \%$ (wt/vol) sucrose solution. Fully fill the syringe pump with $60 \%$ (wt/vol) sucrose. Read a spare $89-\mathrm{mm}$ gradient and adjust the UV detector and recorder sensitivity, as well as the recorder baseline setting, according to the manufacturer's recommendations. The normal recorder sensitivity range for the experiment would be between the 0.2 and 2 sensitivity settings of the absorbance detector. Ensure stable baseline reading in this range.

\section{Procedure}

Expand All

Steps 1 - 25: Steps 1-25: fixation and lysis of cells 
1. Grow the desired yeast cell line from $2-50 \mathrm{ml}$ of starting culture in 1 liter of YPAD medium to an $\mathrm{OD}_{600}$ value of $0.6-0.8$ arbitrary units (a.u.) per 1-cm path. We recommend starting with 1.1 $\mathrm{g}$ of wet cell mass. If you are using other growth conditions, make sure to readjust them accordingly to yield an equivalent amount of cell mass. Do not attempt to harvest more than two cultures in parallel.

Critical step: Media composition may affect subsequent cross-linking efficiency. This protocol was tested with YPAD medium only.

2. Chill four $500-\mathrm{ml}$ polypropylene centrifuge bottles with sealing closures and a 2 liter glass or polypropylene beaker, by placing them in crushed ice in a large container.

Caution: Centrifuge bottles must achieve hermetic sealing.

3. Shortly before harvesting, add $250 \mathrm{~g}$ of crushed ice to the large chilled beaker.

4. Snap-chill the cells by pouring the cell suspension into the large beaker with the ice. Stir vigorously with the polypropylene rod until the ice has completely melted.

Critical step: Proceed immediately to Step 5 when all the ice has melted.

5. Start the timer and add $100 \mathrm{ml}$ of freshly depolymerized $30 \%(\mathrm{wt} / \mathrm{vol})$ paraformaldehyde to the beaker $(2.22 \%$ (wt/vol) final concentration), stirring vigorously with the rod.

Caution: Paraformaldehyde and formaldehyde are harmful. Perform all handling in an appropriate chemical fume hood, in a well-ventilated area. Wear personal protection equipment (gloves, goggles, laboratory coat). Ensure hermetic sealing of the centrifuge tubes for handling outside of the fume hood. Carefully inspect the 500-ml centrifuge tubes for leaks while in the fume hood and after each centrifugation.

Critical step: Steps 5-12 are time-critical. Any variations of time and regimen will affect cross-linking efficiency.

6. Pour the mixture into the centrifuge tubes. Sort into pairs of tubes with equal amounts to help balance the centrifuge later. Incubate the tubes at $4{ }^{\circ} \mathrm{C}$ for $8 \mathrm{~min}$. To adhere to the protocol when using centrifuges requiring longer braking times at Step 7, the 8-min incubation should be shortened as necessary to result in the same processing time between Steps 5 and 12 . During incubation, transfer the tubes to the appropriate chilled $\left(4{ }^{\circ} \mathrm{C}\right)$ centrifuge rotor.

7. Pellet the cells by centrifugation of the tubes at $5,000 \mathrm{~g}$ for $5 \mathrm{~min}$ at $4{ }^{\circ} \mathrm{C}$.

8. Open the tubes and remove the supernatant by gently turning the tubes upside down over a formaldehyde waste container. Place the tubes on the crushed ice.

Caution: Paraformaldehyde and formaldehyde are harmful. Perform all handling in an appropriate chemical fume hood, in a well-ventilated area. Wear personal protection equipment (gloves, goggles, laboratory coat).

9. Immediately add $40 \mathrm{ml}$ of the ice-cold buffer A-1 (containing glycine) to each tube and resuspend the cells thoroughly by pipetting.

Critical step: 


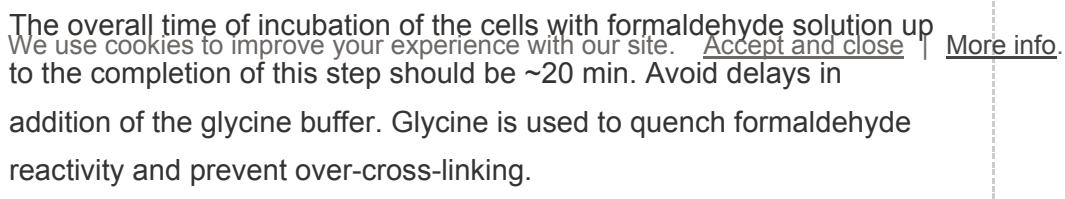

10. Transfer the cell suspension to a preweighed $50-\mathrm{ml}$ conical polypropylene centrifuge tube. Keep the tube on ice.

Caution: Paraformaldehyde and formaldehyde are harmful. Perform all handling in an appropriate chemical fume hood, in a well-ventilated area. Wear personal protection equipment (gloves, goggles, laboratory coat).

11. Pellet the cells at $5,000 \mathrm{~g}$ for $5 \mathrm{~min}$ at $4{ }^{\circ} \mathrm{C}$.

12. Remove the supernatant by gently turning the tubes upside down over a formaldehyde waste container. Use of a chemical fume hood after this step may not be necessary.

13. Resuspend the cell pellet in $40 \mathrm{ml}$ of buffer $\mathrm{A}$ by thorough pipetting.

14. Repeat Steps 11-13 once more.

15. Repeat Step 11 once.

16. Carefully aspirate the cell pellet by gentle pipetting while keeping the tube on ice. Weigh the tube and calculate the wet cell weight. The wet cell weight will normally be $\sim 1.1 \mathrm{~g}$.

17. Resuspend the cell pellet in $40 \%$ (vol/wt) buffer A-2 (i.e., $400 \mu \mathrm{l}$ per $1 \mathrm{~g}$ of cells) by pipetting while keeping the tube on ice.

18. Place a new 50-ml conical polypropylene centrifuge tube vertically into a rack within a thermally insulated container (such as a new polystyrene foam box) filled with liquid nitrogen to a depth of $\sim 3 \mathrm{~cm}$ at most. Fill the tube halfway with clean liquid nitrogen.

Caution: Wear appropriate protective equipment, such as thermally insulated gloves, when handling liquid nitrogen. Ensure that any container used to hold liquid nitrogen does not leak, and that the tube rack inside will not float up or fall on its side. Work in a well-ventilated area to avoid oxygen depletion.

19. Using a 1-ml pipettor, drip the cell suspension (from Step 17) into the tube containing liquid nitrogen. Avoid aggregation of partially frozen droplets by dripping slowly, allowing the droplets to freeze completely before adding new ones.

20. Transfer the tube with the frozen cell suspension droplets to room temperature and wait until the liquid nitrogen evaporates completely. Seal the tube with its cap and place it back in the container with the liquid nitrogen.

Caution: Ensure that the liquid nitrogen is completely evaporated before sealing. Leftover liquid nitrogen in a sealed tube can cause a hazardous pressure build-up.

Pause point: The frozen cells can be stored at $-80^{\circ} \mathrm{C}$ or below for at least 6 months. 
21. Assemble a stainless-steel grinding canister suitable for 1 - to $3-m l$ loads (i.e., Retsch $10-m$ - 10 l stainless steel jar with two 12-mm stainless steel grinding balls) and precool it by submerging in liquid nitrogen.

Caution: Ensure that the grinding canister sealing O-ring is clean and intact. Seal the canister tightly to avoid liquid nitrogen leaking inside. Wear appropriate protective equipment, such as thermally insulated gloves, when handling liquid nitrogen. Work in a well-ventilated area to avoid oxygen depletion.

22. Quickly transfer the frozen cell suspension droplets to the grinding canister, seal the canister and recool it by submerging it in liquid nitrogen until intensive boiling ceases.

23. Shake the cooled canister in a mill at $27 \mathrm{~Hz}$ for $1 \mathrm{~min}$.

Critical step: Always balance the grinding canister with another one of the same type, even if the sample requires only one canister for processing.

24. Recool the canister as in Step 21 and repeat Step 23 one more time.

25. Open the grinding canister and partially submerge its reservoir part in liquid nitrogen in a thermally insulated (e.g., polystyrene foam) container to avoid heating. Precool a suitable plastic or stainless steel spatula and at least two preweighed nuclease-free polypropylene 1.5-ml microcentrifuge tubes by partial submersion in the liquid nitrogen. Transfer the ground cell suspension powder to the tubes.

Critical step: Avoid static electricity and intense airflows during the cell powder transfer to avoid sample loss.

Pause point: The closed tubes with the frozen ground cell suspension can be stored at $-80{ }^{\circ} \mathrm{C}$ or below for at least 6 months.

Steps 26 - 72: Steps 26-72: isolation of translation complexes and mRNA footprints

Timing: $\sim 3 \mathrm{~d}$

26. Prepare two $35-\mathrm{mm}, 10-20 \%$ (wt/vol) sucrose gradients as described in Box 1 and keep them at $4{ }^{\circ} \mathrm{C}$ for $1-3 \mathrm{~h}$ before use.

27. Thaw two tubes $(2 \times 500 \mathrm{mg})$ of the frozen ground cell suspension from Step 25 by placing the tubes at room temperature with occasional vortexing. Monitor the thawing closely.

28. As soon as the last pieces of the lysate thaw, transfer the tubes to ice, and then add $3.6 \mu \mathrm{l}$ of RNaseOUT (40 U per $100 \mathrm{mg}$ of wet cell mass)

29. Vortex at high speed for a further $10 \mathrm{~s}$ and then immediately put the tubes on the ice. Perform all the subsequent operations on crushed ice unless otherwise indicated.

30. Pellet cell debris by centrifugation of the tubes at $13,500 \mathrm{~g}$ for $5 \mathrm{~min}$ at $4{ }^{\circ} \mathrm{C}$.

31. Carefully transfer the supernatants to a new $1.5-\mathrm{ml}$ microcentrifuge tube. Avoid disturbing the pellets.

32. Pellet cell debris by centrifugation of the tube at $13,500 \mathrm{~g}$ for $10 \mathrm{~min}$ at $4{ }^{\circ} \mathrm{C}$.

33. Repeat Step 31. The clarified lysate volume should be $\sim 400 \mu \mathrm{l}$. 


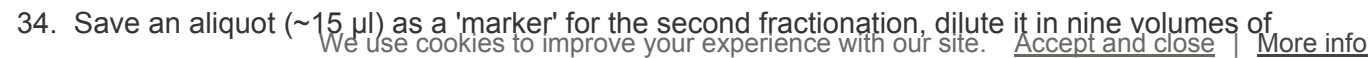
cold buffer A-3 and place it on ice (used at Steps 49 and 55).

Critical step: Check that lysates from Step 33 are buoyant in 10\% (wt/vol) sucrose before proceeding, by gently pipetting a small aliquot $(\sim 2 \mu \mathrm{l})$ of lysate into a spare tube of $10 \%(\mathrm{wt} / \mathrm{vol})$ sucrose. If the lysate sinks, dilute it with buffer B until it is buoyant.

35. Gently layer half of the clarified cell lysate from Step $33(\sim 200 \mu \mathrm{l})$ onto each of the two 35-mm gradients. Use a 200- $\mu$ l pipettor or equivalent and slowly dispense the lysate, with the pipettor tip contacting the tube wall perpendicularly, and allow the solution to flow down the tube wall without causing turbulence.

36. Equalize the tube weights using appropriate analytical balances and addition of buffer B atop of the gradients, similar to the addition of the sample as described in Step 35.

Critical step: If spare balance tubes are needed, equalize their weight and weight distribution with the sample-containing tubes. Use spare sucrose gradients overlaid with an amount of buffer equivalent to the sample overlay and not tubes with uniform sucrose concentration.

37. Centrifuge the tubes in a TLS-55 rotor at 52,000 r.p.m. for 85 min at $4{ }^{\circ} \mathrm{C}$. At these conditions, the rotor $k$ factor is 55.7 , RCFmax is $231,679 \mathrm{~g}$ and RCFavg is $179,740 \mathrm{~g}$. Adjust the conditions accordingly if you are using another rotor configuration.

Critical step: Centrifugation conditions were optimized for the buffer type, sucrose density, temperature and type of rotor presented here. If any of these conditions are altered, results may be unpredictable unless further optimization is carried out

38. Decant the supernatant by gently turning the tubes upside down, and place the tubes back on ice. The pellet will appear slightly opaque, with a jelly-like consistency, and it is not easily dislodged.

39. Add $1 \mathrm{ml}$ of buffer A-3 to each tube by slowly dispensing around the tube wall. Avoid direct pouring on the pellet.

40. Wash the pellet and tube walls by vortexing the tube slowly (50-100 r.p.m.) for $3 \mathrm{~s}$

41. Remove the supernatant by tilting the tube and pipetting the solution out.

42. Add $200 \mu \mathrm{l}$ of buffer A-3 to each tube and resuspend the pellet by intensive pipetting and scraping the pellet with pipettor tip, if necessary.

43. Transfer the resultant suspension to a new nuclease-free polypropylene 1.5-ml microcentrifuge tube and keep it on ice.

44. Bring residual pellet fragments to the bottom of the tube by centrifuging it at $13,500 \mathrm{~g}$ for $5 \mathrm{~min}$ at $4{ }^{\circ} \mathrm{C}$.

45. Using a microcentrifuge tube pestle, mash the pellet fragments thoroughly while keeping the tube on ice.

46. Vortex the tube at high speed for $2 \min$ at $4{ }^{\circ} \mathrm{C}$.

47. Clarify the mixture by centrifugation of the tube at $13,500 \mathrm{~g}$ for 5 min at $4{ }^{\circ} \mathrm{C}$. 
48. Transfer the supernatant to a new nuclease-free polypropylene 1.5 -ml microcentrifuge tube.

49. Measure the OD of the polysome suspension at the 260-nm wavelength versus that of buffer A-3, using a NanoDrop spectrophotometer or equivalent. The OD will typically be 150-300 a.u. per $1-\mathrm{cm}$ path at $260 \mathrm{~nm}$. In addition, measure the diluted lysate aliquot from Step 34, which will typically be $40-70$ a.u. per $1-\mathrm{cm}$ path at $260 \mathrm{~nm}$.

\section{Troubleshooting}

50. If construction of a control total input (total translated RNA) library is desired, preserve a 15- to $30-\mu \mathrm{l}$ aliquot of the polysomes by flash-freezing it in a separate microcentrifuge tube in liquid nitrogen and storing at $-80^{\circ} \mathrm{C}$. The total translated RNA can be deblocked from the cross-links using the same approach as described for the SSU and complete ribosome libraries in Steps 63-72, followed by rRNA depletion with a RiboMinus Eukaryote Kit and nonspecific fragmentation using a NEBNext Magnesium RNA Fragmentation Module (strictly according to the manufacturer's recommendations and using incubation at $94{ }^{\circ} \mathrm{C}$ for 6 min during fragmentation). The total translated RNA library can further be processed in parallel with the SSU and complete ribosome library construction starting from Step 73.

Pause point: Flash-freeze both the polysomes and marker aliquot from Step 34 in liquid nitrogen; they can be stored at $-80^{\circ} \mathrm{C}$ or below for at least 6 months.

51. Prepare two $7.5-30 \%$ (wt/vol) gradients of sucrose in buffer B using two thin-walled polypropylene $89 \times 14-\mathrm{mm}$ centrifuge tubes (Beckman-Coulter), as described in Box 1, and keep them at $4{ }^{\circ} \mathrm{C}$.

52. Add $15 U$ of $E$. coli RNase I per 1 a.u. of the solution at the $260-n m$ wavelength to digest the unprotected RNA. Dilute RNase I with buffer A-3 if the stock solution is too concentrated for precise pipetting.

Critical step: It is critical to adhere to the recommended conditions of digestion and carefully measure the amount of the added RNase I. The RNase I unit referred to here is that originally used by Ambion and is defined as the amount of the enzyme required to produce $1 \mu \mathrm{g}$ of acid-soluble material from mouse liver RNA in $30 \mathrm{~min}$ at $37^{\circ} \mathrm{C}$. RNase I batches may have undocumented variations in activity and may require experimentation to achieve optimal digestion conditions.

53. Incubate the digestion mixture for $45 \mathrm{~min}$ at $23^{\circ} \mathrm{C}$.

Critical step: Expect variable results if the digestion conditions are altered. It is critical to proceed with the subsequent steps immediately after the completion of the digestion, as the RNase I digestion continues until the target complexes are fractionated away from the ribonuclease at Step 58.

54. Load the digestion mixture $(\sim 200 \mu \mathrm{l})$ onto one of the $89-\mathrm{mm}$ gradients. Use the same approach for loading as described in Step 35.

55. Dilute the aliquot of the original cell lysate set aside in Step 34 with buffer $B$ to the same volume as that of the digestion mixture $(\sim 200 \mu \mathrm{l})$.

56. Overlay the diluted cell lysate onto the unused gradient, to be used both as a balance for ultracentrifugation and as a sedimentation 'marker' to give the approximate elution volume for the SSU during fractionation. Use the same approach for loading, as described in Step 35. 
57. Equalize tube weights using appropriate balances, overlaying additional buffer B on the marker
More info gradient if needed.

Critical step: If spare balance tubes are needed, equalize their weight and weight distribution with the sample-containing tubes. Use spare sucrose gradients overlaid with an amount of buffer equivalent to that of the sample overlay and not tubes with uniform sucrose concentration.

58. Centrifuge the tubes in an SW-41 Ti rotor at 38,000 r.p.m. for $4 \mathrm{~h}$ at $4{ }^{\circ} \mathrm{C}$. Under these conditions, the rotor $k$ factor is 143.9 , RCFmax is $247,606 g$ and RCFavg is $178,305 g$. Adjust the conditions accordingly if you are using another rotor configuration.

59. Prepare a Brandel gradient fractionator or a functionally equivalent setup in a cold room. Use a 254-nm-wavelength UV filter, a 2-0.5 absorbance detection range and a $10 \mathrm{~mm} / \mathrm{min}$ paper feed speed (see Fig. 5b for an example and suggested points of sensitivity switching).

60. Adjust the baseline for UV absorbance by either using $20 \%$ (wt/vol) sucrose in buffer B solution or recording the actual absorbance profile of a spare empty $14 \times 89-\mathrm{mm}$ gradient using a 1.5 $\mathrm{ml} / \mathrm{min}$ flow speed.

Critical step: The large difference between the absorbance of the SSU $\sim 40$ S and complete ribosome $\sim 80 S$ peaks usually requires switching the sensitivity of the UV absorbance detector during fractionation to visualize each of them conveniently. Consult the user manual on how to adjust the settings in real time to ensure that the baseline does not change substantially when switching the sensitivity. This can be verified by loading a blank sample into the absorbance cell and observing the baseline absorbance reading while switching the sensitivity setting between 2.0 and 0.2 on the Brandel fractionator.

61. Fractionate the gradients at $1.5 \mathrm{ml} / \mathrm{min}$. Fraction volumes are typically $1.5 \mathrm{ml}$ for SSUs ( 40S) and $2 \mathrm{ml}$ for ribosomes ( 80S). If the absorbance profiles are not yet known, follow option A. For known profiles, follow option B.

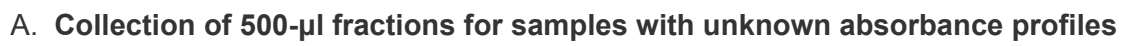

i. Collect $500-\mu l$ fractions.

ii. Identify SSU ( 40S) and ribosome ( 80S) peaks.

iii. Pool fractions of the same type.

\section{B. In-line collection for samples with known absorbance profiles}

i. Collect SSU and ribosome peaks, allowing a sufficient time delay between peak observation and peak collection for displacement of the internal tubing volume between the detector cuvette and the collection point (see Fig. $5 \mathrm{~b}$ for an example of the offset fraction collection).

Critical step: We recommend fractionating the marker sample (set up in Step $56)$ first, in order to identify the position of the SSU ( 40S) and ribosome ( 80S) peaks in the gradient. Marking the distance between the top of the gradient and these peaks will aid in the later collection of the equivalent fractions from the TCP-seq gradients. For gradients loaded with between 25 and $50 \mathrm{OD}_{260}$ units, the sensitivity setting of the UV reader can be set to 2.0 to 


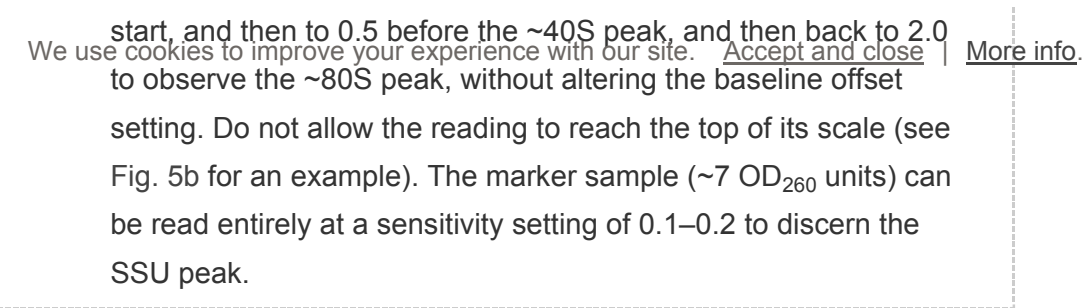

\section{$\underline{\text { Troubleshooting }}$}

62. Transfer no more than $350 \mu$ l of the mixed SSU and ribosome fractions to new low-binding nuclease-free polypropylene 1.5-ml microcentrifuge tubes with locking devices (Eppendorf Safe-Lock or equivalent).

Pause point: Flash-freeze the resultant solutions in liquid nitrogen; the tubes can be stored at $-80^{\circ} \mathrm{C}$ or below for at least 6 months.

63. Select appropriate volumes of the SSU and complete ribosome fractions from Step 61 and the total input fraction from Step 50. The required volumes will depend on the desired amplification and sequencing depths of the libraries derived from each of these fractions. Generally, use the whole volume of the SSU ( 40S), $\sim 0.02$ volumes of the SSU fraction volume for the complete ribosome ( $\sim 80 \mathrm{~S}$ ), and $\sim 0.003$ volumes of the SSU fraction volume for the total translated mRNA (total input, from Step 50) fractions to obtain roughly comparable numbers of protected mRNA fragment reads.

64. Add stop solution to $10 \%$ (vol/vol) $(40 \mu \mathrm{l} 100 \%)$, Tris- $\mathrm{HCl}\left(\mathrm{pH} 7.4\right.$ at $\left.25^{\circ} \mathrm{C}\right)$ to $10 \mathrm{mM}(4 \mu \mathrm{l}, 1$ $\mathrm{M})$, glycine to $10 \mathrm{mM}(1.6 \mu \mathrm{l}, 2.5 \mathrm{M})$ and deionized nuclease-free water $(4.4 \mu \mathrm{l}$ if the starting fraction volume was $350 \mu \mathrm{l}$ ) to obtain $400 \mu \mathrm{l}$ as the final volume.

65. Add $400 \mu \mathrm{l}$ of acidic phenol/chloroform solution and vigorously shake the mixtures using a vortex set to maximum speed for 2 min.

Caution: Phenol and chloroform are corrosive and toxic. Avoid physical contact with liquids and work in a well-ventilated area or under a fume hood. Always use at least gloves and protective goggles or a face shield when working with phenol or chloroform.

66. Place the tubes with the mixtures into a thermomixer and continue shaking at high speed $\left(1,300\right.$ r.p.m.) for $45 \mathrm{~min}$ at $65^{\circ} \mathrm{C}$ to reverse the cross-links.

Critical step: There will be a small pressure build-up in the tubes due to heating and accumulation of organic vapors, and tubes without locking mechanisms may leak or open unexpectedly. For this reason, it is preferable to use a thermomixer, in which the tubes are fully enclosed in the thermoblock.

67. Centrifuge the tubes with the mixtures at $15,000 \mathrm{~g}$ for $5 \mathrm{~min}$ at room temperature to facilitate separation of the phases.

68. Carefully transfer the aqueous phases to the new $1.5-\mathrm{ml}$ microcentrifuge tubes. To avoid crosscontamination, do not attempt to recover the aqueous phases completely. A reasonable recovery volume is $300-350 \mu \mathrm{l}$.

69. Perform ethanol precipitation of nucleic acids as outlined in Box 2.

Box 2: DNA or RNA cleanup using ethanol precipitation • TIMING $\sim 4 \mathrm{~h}$ 
We use cookies to improve your experience with our site. Accept and close | More info.

Full box

70. Resuspend the dried pellets in $30 \mu \mathrm{l}$ of HE buffer.

71. Estimate the concentration of nucleic acids and overall quality of the sample by measuring UV absorbance versus that of the corresponding buffer. For the SSU fraction, the procedure will normally result in 1-2 $\mu \mathrm{g}$ of single-stranded RNA with an $\mathrm{OD}_{260}$ to $\mathrm{OD}_{280}$ ratio of $\sim 2.00$.

Critical step: Ensure the absence of phenol in the samples, as subsequent steps require efficient enzymatic processing.

\section{Troubleshooting}

72. If you are performing the procedure for the first time, we recommend assessing RNA sample fragmentation by electrophoretic separation and identifying average fragments sizes to relate nucleic acid mass and molarity for each sample. Use an Agilent 2100 Bioanalyzer equipped with an RNA 6000 Pico Kit according to the manufacturer's recommendations, or use a functionally equivalent setup to achieve this.

Pause point: The resultant RNA solution can be stored at $-80^{\circ} \mathrm{C}$ or below for at least 6 months.

Steps 73 - 108: Steps 73-108: conversion of the RNA fragments to a circularized cDNA library

Timing: $\sim 2 \mathrm{~d}$

73. Use $\sim 1 \mu \mathrm{g}$ of RNA derived from the SSU fraction (0.5-1 volumes of SSU RNA from Step 70) and $2 \mu \mathrm{g}$ of RNA derived from the complete ribosome fraction. This will normally equal to $\sim 8$ pmol of RNA fragments with average fragment length of $350 \mathrm{nt}$ for the SSU fraction.

Critical step: All estimates of sample concentration in this protocol are based on the equivalent proportion of starting material represented in the sample, rather than on direct measurement. Estimation of nucleic acid molarity by spectrometry will no longer yield accurate results after polyadenylation (Step 81)

Critical step: All steps before library amplification (Step 122) should be conducted in a 'pre-PCR' area away from areas where the amplified library products are handled.

Critical step: For Steps 73-122, in which the cDNA library is amplified, it is highly recommended to use tubes with low affinity for nucleic acids (e.g., Eppendorf DNA LoBind tubes or equivalent) to reduce material loss.

74. Transfer $15 \mu \mathrm{l}$ of the RNA solution from Step 70 (or another desired amount as determined in Step 72 ) to a new low-binding $0.5-\mathrm{ml}$ microcentrifuge tube.

75. Heat the tube in a thermoblock for $2 \mathrm{~min}$ at $70^{\circ} \mathrm{C}$ and immediately transfer it to ice. 
76. Incubate the tube for $5 \mathrm{~min}$ on ice.

77. Add the following components to the tube (containing $15 \mu \mathrm{l}$ of the RNA fragment mixture) in the order listed to perform the end-repair reaction (final volume: $20 \mu \mathrm{l}$, final concentration of RNA: $\sim 0.4 \mathrm{pmol} / \mu \mathrm{l})$.

78. Incubate the reaction mixture for $2 \mathrm{~h}$ at $37^{\circ} \mathrm{C}$.

79. Add $14.5 \mu \mathrm{l}$ of deionized, nuclease-free water and mix.

80. Heat the tube at $65{ }^{\circ} \mathrm{C}$ for $20 \mathrm{~min}$ in a thermoblock to inactivate T4 PNK.

81. Place the tube on ice immediately, and add the following components to the tube (containing $34.5 \mu \mathrm{l}$ of the end-repaired RNA mixture) in the order listed and mix to perform 3' polyadenylation. The final concentration of RNA will be $\sim 0.16 \mathrm{pmol} / \mu \mathrm{l}$.

82. Incubate the reaction mixture $(50 \mu \mathrm{l})$ for $1 \mathrm{~h}$ at $37^{\circ} \mathrm{C}$

\section{Troubleshooting}

83. Add $5 \mu \mathrm{l}$ of stop solution and $50 \mu \mathrm{l}$ of acidic phenol/chloroform solution and vigorously shake the mixture for 2 min using a vortex set to maximum speed.

Caution: Phenol and chloroform may be harmful. Avoid physical contact with liquids and work in a well-ventilated area or under a fume hood. Always use at least gloves and protective goggles or a face shield when working with phenol or chloroform.

84. Centrifuge the tube at $12,000 \mathrm{~g}$ for $5 \mathrm{~min}$ at room temperature.

85. Recover the upper aqueous phase using a 100 - to $200-\mu l$ pipettor equipped with a fine tip. To avoid contamination, do not attempt to recover the solution completely; leave $5-10 \mu$ l of the aqueous phase in the tube instead.

86. Perform gel filtration of the sample as outlined in Box 3 .

Box 3: Gel filtration of small volumes of DNA and RNA solutions • TIMING 15 min

Full box

Pause point: The gel-filtered RNA sample can be stored at $-80^{\circ} \mathrm{C}$ or below for at least 6 months.

87. Transfer $25 \mu \mathrm{l}$ of the gel-filtered sample ( 4 pmol or half of the original RNA fragments, if the recovery volume in Step 86 was $50 \mu \mathrm{l}$ ) to a new $200-\mu$ l thin-walled low-binding microcentrifuge tube.

Pause point: The other half of the sample can be stored at $-80^{\circ} \mathrm{C}$ or below for at least 6 months as a backup. 
88. Assemble and mix the following components in the tube (containing $25 \mu$ lof the gel-filtered RNA solution) in the order listed to prepare an annealed template for the reverse transcription reaction. The final concentration of RNA (at Step 91) will be $\sim 0.09 \mathrm{pmol} / \mu \mathrm{l}$.

89. Heat the mixture $(38.25 \mu \mathrm{l})$ in a heated lid thermocycler at $75^{\circ} \mathrm{C}$ for $3 \mathrm{~min}$ to denature the RNA and the primer.

90. Cool the mixture to $65^{\circ} \mathrm{C}$ and slow-ramp $\left(3^{\circ} \mathrm{C}\right.$ per min) the temperature to $55^{\circ} \mathrm{C}$ to anneal the RNA and the primer.

91. While keeping the tube in the thermocycler, quickly add and mix by pipetting the following components in the order listed to initiate the reverse transcription reaction (final volume: $45 \mu \mathrm{l}$ ).

Critical step: Avoid prolonged incubation of the tube during addition of the components. Open the tube only when you add or mix components, and immediately close it upon completion. Do not allow the tube to cool.

92. Slow-ramp $\left(3^{\circ} \mathrm{C}\right.$ per min) the temperature of the mixture to $50^{\circ} \mathrm{C}$ and incubate further at $50{ }^{\circ} \mathrm{C}$ for $30 \mathrm{~min}$

93. Heat the tube in the thermocycler to $60{ }^{\circ} \mathrm{C}$ and incubate at $60{ }^{\circ} \mathrm{C}$ for $5 \mathrm{~min}$ to denature residual primer-template hybrids.

94. Place the tube on ice for 2 min.

95. Add $20 \mathrm{U}$ of $E$. coli exonuclease I ( $1 \mu \mathrm{l}$ of $20 \mathrm{U} / \mu \mathrm{l}$ Exo I) and mix by brief vortexing or pipetting.

96. Incubate in the thermocycler for $20 \mathrm{~min}$ at $37^{\circ} \mathrm{C}$.

97. Add EDTA to $23 \mathrm{mM}\left(2.21 \mu \mathrm{l}\right.$ of $500 \mathrm{mM}$ EDTA, pH 8.0 at $\left.25^{\circ} \mathrm{C}\right)$ and mix by vortexing or pipetting to stop Exo $\mathrm{I}$.

98. Transfer the tube from the thermocycler to a dry-heat oven set at $60^{\circ} \mathrm{C}$.

Critical step: The $60{ }^{\circ} \mathrm{C}$ heated oven is used to prevent the unextended reverse transcription split-adaptor primer from co-purifying with the synthesized cDNA. However, because efficiency of cDNA purification may depend on the integrity of the RNA template to which it remains attached, do not keep the tube at $60^{\circ} \mathrm{C}$ for an unnecessarily long time. Immediately proceed to Step 99

99. Purify the synthesized cDNA using AMPure XP beads as outlined in Box 4, but using a dryheat oven set at $60^{\circ} \mathrm{C}$ for all procedures and keeping all reagents preheated to $60{ }^{\circ} \mathrm{C}$ at steps $6-10$ of Box 4 to prevent co-purification of the unextended primer. Use $20 \mu \mathrm{l}$ of HE buffer to elute the cDNA

Box 4: Purification of DNA using AMPure XP beads • TIMING $\sim 1 \mathrm{~h}$

Full box 
100. Heat the tube with all the purified cDNA $(20 \mu \mathrm{u})$ from Step $99 \mathrm{for} 10 \mathrm{~min}$ at $80{ }^{\circ} \mathrm{C}$ to denature nucleic acids.

101. Place the tube on ice and incubate for $3 \mathrm{~min}$.

102. Add $1 \mu \mathrm{l}$ of RNase $A / T$ mix and mix by brief vortexing or pipetting.

103. Incubate the tube for $20 \mathrm{~min}$ at $37^{\circ} \mathrm{C}$ to digest the RNA contaminants. Transfer $10 \mu \mathrm{l}$ of the sample (cDNA equivalent to $2 \mathrm{pmol}$ of the original RNA) to a new thin-wall low-binding 200- $\mu \mathrm{l}$ microcentrifuge tube. Store the residual mixture as a backup at $-20^{\circ} \mathrm{C}$ or below for 6 months.

Pause point: Samples can be frozen and stored $-20^{\circ} \mathrm{C}$ or below for at least 6 months.

104. Assemble and mix the following components in the tube (containing $10 \mu \mathrm{l}$ of the cDNA solution) in the order listed to initiate the cDNA circularization reaction (final volume: $20 \mu$, final concentration of cDNA: $\sim 0.1 \mathrm{pmol} / \mu \mathrm{l})$.

Critical step: We recommend assembling a parallel reaction in which cDNA is replaced by an equivalent amount of the split-adaptor primer to generate an insert-less circular template. This can be used later to precisely identify the size-selection cutoff of the library. Treat this sample in parallel up to Step 107 and use it in a complementary amplification reaction and as a control in Steps 121-128.

105. Incubate the tube for $5 \mathrm{~h}$ at $60{ }^{\circ} \mathrm{C}$ in the thermocycler.

106. Add EDTA to $23.8 \mathrm{mM}\left(1 \mu \mathrm{l}\right.$ of $500 \mathrm{mM}$ EDTA, $\mathrm{pH} 8.0$ at $\left.25^{\circ} \mathrm{C}\right)$ and mix by brief vortexing or pipetting to stop the reaction.

107. Heat the tube to $80^{\circ} \mathrm{C}$ in the thermocycler for $10 \mathrm{~min}$ to inactivate CircLigase II.

108. Cool the sample to room temperature for $3 \mathrm{~min}$, and purify the cDNA using AMPure XP beads, as outlined in Box 4, but use $10 \mu \mathrm{l}$ of HE buffer to elute the cDNA at stage 17.

Pause point: Samples can be frozen and stored at $-20^{\circ} \mathrm{C}$ or below for at least 6 months.

Steps 109 - 120: Steps 109-120: depletion of the unwanted rRNA sequences

Timing: $\sim 1 \mathrm{~d}$

109. Premix the following components in the order listed in a new thin-walled low-binding $200-\mu l$ microcentrifuge tube.

110. Heat the tube in a thermocycler at $95^{\circ} \mathrm{C}$ for $3 \mathrm{~min}$.

111. Slow-ramp the thermocycler temperature $\left(3^{\circ} \mathrm{C} / \mathrm{min}\right)$ to $75^{\circ} \mathrm{C}$.

112. While keeping the tube in the thermocycler, quickly add $2 \mu$ of $10 \times$ DSN buffer by pipetting and mix.

Critical step: Do not remove the tube from the thermocycler, and do not allow the contents of the tube to cool. Do not keep the tube open longer than it takes to add and mix components.

113. Slow-ramp the thermocycler temperature $\left(3^{\circ} \mathrm{C} / \mathrm{min}\right)$ to $60^{\circ} \mathrm{C}$ and incubate for $1 \mathrm{~min}$ at $60^{\circ} \mathrm{C}$. 


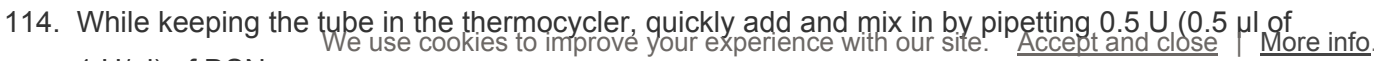
$1 \mathrm{U} / \mathrm{\mu l})$ of DSN.

Critical step: Do not remove the tube from the thermocycler, and do not allow the contents of the tube to cool. Do not keep the tube open longer than it takes to add and mix components.

115. Slow-ramp the thermocycler temperature $\left(3^{\circ} \mathrm{C} / \mathrm{min}\right)$ to $48^{\circ} \mathrm{C}$ and incubate for 20 min at $48^{\circ} \mathrm{C}$.

116. While keeping the tube in the thermocycler at $48^{\circ} \mathrm{C}$, add $0.75 \mu \mathrm{l}$ of $500 \mathrm{mM}$ EDTA $(\mathrm{pH} 8.0$ at $25^{\circ} \mathrm{C}$ ) and $54.25 \mu \mathrm{l}$ of HE buffer and mix by pipetting to stop the reaction.

Critical step: Do not remove the tube from the thermocycler, and do not allow the contents of the tube to cool.

117. Remove the tube from the thermocycler and add $75 \mu \mathrm{l}$ of phenol/chloroform/isoamyl alcohol with a neutral $\mathrm{pH}$.

Caution: Phenol and chloroform may be harmful. Avoid physical contact with liquids and work in a well-ventilated area or under a fume hood.

Always use at least gloves and protective goggles or a face shield when you are working with phenol or chloroform.

118. Shake the tube using a vortex set at a high r.p.m. for 2 min at room temperature.

119. Centrifuge the tube at $12,000 \mathrm{~g}$ for $10 \mathrm{~min}$ at room temperature.

120. Using a 100- to $200-\mu \mathrm{l}$ pipettor equipped with a fine tip, remove $50-65 \mu \mathrm{l}$ of the aqueous phase from the mixture and subject it to gel filtration as outlined in Box 3. You may wish to replace the MicroSpin G-25 column or its equivalent with a MicroSpin G-50 or similar having a higher molecular-weight cutoff. The cutoff should be $<50$ nt of linear single-stranded DNA.

Critical step: Do not attempt to remove the entire aqueous phase, in order to avoid contamination.

Pause point: The resultant solution can be stored at $-20^{\circ} \mathrm{C}$ or below for at least 6 months.

Steps 121 - 141: Steps 121-141: amplification, size selection and sequencing of the cDNA library

Timing: $\sim 5 \mathrm{~d}$

121. Assemble and mix the following components in a new thin-walled low-binding 200- $\mu \mathrm{l}$ microcentrifuge tube in the order listed to amplify the gel-filtered cDNA. Use half of the sample from Step 120 at most, keeping the rest of it as a backup. Primer sequences can be found in Supplementary Table 1.

Critical step: We recommend setting up a parallel reaction with empty split-adaptor primer ligation products from Step 104, and running this sample in parallel.

122. Use the following settings to program the thermocycler to amplify the cDNA.

Critical step: After amplification, do not return the samples to the dedicated pre-PCR area. 
We use-cookies to-improve-your experience-with-our -site:-- Accept-and close-- - More info

Critical step: Optimizing the amplification depth may be required. A test

electrophoretic analysis of a small (3-5 $\mu \mathrm{l})$ volume of the amplification

mixture can be performed after 12 cycles using conditions resembling

preparative separation (outlined below in Steps 123-127). The amplified

material should be clearly visible to enable size selection.

\section{Troubleshooting}

Pause point: The amplified cDNA can be stored at $-20^{\circ} \mathrm{C}$ or below for at least 6 months.

123. Mix the amplified cDNA solution with $5 \mu \mathrm{l}$ of $6 \times$ GRGreen Loading Buffer by pipetting.

124. To make an electrophoretic mobility marker, assemble and mix by pipetting the following into a new 1.5-ml microcentrifuge tube.

Critical step: We recommend also including 1-2 $\mu$ of the amplification reaction mixture from the parallel reaction made using the empty split-adaptor reverse transcription primer ligation product as a template (see Steps 104 and 121), replacing an equivalent volume of deionized water.

125. Load all the mixture from Step 123 and $30 \mu \mathrm{l}$ of the mixture from Step 124 into two adjacent $1 \times$ $10 \times 5$-mm wells (or equivalent) of at least $2.5 \%$ (wt/vol), $8 \mathrm{~cm}$ or more long, 7 -mm-thick native agarose gel made with $0.5 \times$ TBE buffer.

Critical step: Avoid cross-contamination between wells. Use high-resolution and highpurity agarose.

126. Run electrophoretic separation in $0.5 \times$ TBE buffer at $3 \mathrm{~V} / \mathrm{cm}$ for the first $15 \mathrm{~min}, 5 \mathrm{~V} / \mathrm{cm}$ for the next $30 \mathrm{~min}$ and $8 \mathrm{~V} / \mathrm{cm}$ for $1-3 \mathrm{~h}$.

Critical step: Verify the position of the bands on the gel every 45 min by visualizing the gel on a blue-light transilluminator equipped with an appropriate filter. Do not allow the 100-bp band to escape the gel.

127. Stop the separation when the $100-\mathrm{bp}$ band is $0.5-1 \mathrm{~cm}$ away from the anode side of the gel.

\section{Troubleshooting}

128. Using a sharp, clean DNase-free scalpel and a blue-light transilluminator to visualize the gel, excise the region of the amplified cDNA lane between the position of the amplified empty reverse transcription split-adaptor primer band ( 140 bp) —but not including material in that region-and the position of 400-bp DNA band, as anticipated from the control lane.

Critical step: It is important to include fragments that appear to move slower than the empty reverse transcription split-adaptor primer band by as little as 10 $-15 \mathrm{bp}$. This is necessary because insert sequences of $17 \mathrm{nt}$ and more can be reliably mapped to yeast transcriptome and represent functional footprints in the SSU libraries.

129. Transfer the gel to a new $1-\mathrm{ml}$ syringe without a needle attached. 
130. Using the syringe plunger, squeeze the gel into a new Quantum Prep Freeze 'N Squeeze PNA Gel Extraction Spin Column filter assembly (or equivalent $0.45-\mu \mathrm{m}$ filter setup) and keep it at $-20^{\circ} \mathrm{C}$ for at least $30 \mathrm{~min}$.

131. Centrifuge the filter assembly with the gel at $12,000 \mathrm{~g}$ for $10 \mathrm{~min}$ at room temperature.

132. Transfer the collected eluate to a new $1.5-\mathrm{ml}$ microcentrifuge tube.

133. Add $300 \mathrm{mM}$ sodium acetate $\left(\mathrm{pH} 8.0\right.$ at $\left.25^{\circ} \mathrm{C}\right)$ to the gel filter top chamber and shake it with the residual gel pieces at 1,000 r.p.m. for $30 \mathrm{~min}$ at room temperature.

134. Repeat Steps 131 and 132.

135. Combine and mix the eluates from Steps 132 and 134 and distribute in $350-\mu l$ aliquots in new $1.5-\mathrm{ml}$ low-binding microcentrifuge tubes.

136. Add $350 \mu \mathrm{l}$ of the phenol/chloroform/isoamyl alcohol mixture $\left(\mathrm{pH} 8.0\right.$ at $\left.25^{\circ} \mathrm{C}\right)$ to each tube and shake the tubes using a vortex at high r.p.m. for $2 \mathrm{~min}$.

Caution: Phenol and chloroform may be harmful. Avoid physical contact with liquids and work in a well-ventilated area or under a fume hood. Always use at least gloves and protective goggles or a face shield when working with phenol or chloroform.

137. Centrifuge the tubes at $12,000 \mathrm{~g}$ for $10 \mathrm{~min}$ at room temperature.

138. Using a 100 - to $200-\mu l$ pipettor equipped with a fine tip, carefully transfer $300 \mu \mathrm{l}$ of the upper aqueous phases to new 1.5 low-binding microcentrifuge tubes.

Critical step: Do not attempt to transfer all the aqueous phase, in order to avoid contamination.

139. Clean up the DNA by ethanol precipitation as described in Box 2. Use 15-20 $\mu$ of HE buffer to resolubilize the DNA.

Critical step: We highly recommend analyzing the electrophoretic profile of the amplified cDNA library at this stage and estimating the median insert size and molarity of the sample. Use an Agilent Expert 2100 Bioanalyzer equipped with a high-sensitivity DNA chip or a similar setup to achieve this.

\section{Troubleshooting}

Pause point: The amplified cDNA can be stored at $-20^{\circ} \mathrm{C}$ or below for at least 6 months.

140. Adjust the cDNA library solution molarity to $2 \mathrm{nM}$ using deionized water. If multiplexing libraries between the lanes, adjust to a combined $2 \mathrm{nM}$ concentration of all the molecules per each lane.

141. Sequence the library on an Illumina HighSeq 2500 (or equivalent) strictly according to the manufacturer's instructions provided for libraries generated with a TrueSeq Small RNA Sample Prep Kit. Use 150-nt single-end reads and read depth of at least $50 \mathrm{M}$ per library type.

Steps 142 - 144: Steps 142-144: setup of the virtual machine for data analysis 
142. Create a new, empty directory and set up the TCP-seq virtual machine (YM), which contains preindexed references for aligning and all software required for the completion of this pipeline.

mkdir TCP_VM;

Cd TCP_VM;

vagrant init ।

http://bioinformatics.erc.monash.edu/TCPseq/vm/TCPseq.box;

vagrant up;

143. Create a folder for the sequence data and then log in to the VM. The Bash code for all following steps in this pipeline can be found in the file /TCPseq/pipeline_summary.sh after logging on to the downloaded VM.

mkdir input_data;

vagrant ssh;

pwd ;

Critical step: The directory returned by the pwd command is the location in the VM from which all code blocks below should be executed.

144. (Optional) Get updates, if available. Remember to check the readme files for updates to the protocol given below. From the VM, apply commands as outlined in Box 5 to download reference and annotation data for the pipeline if a different reference genome is desired.

Box 5: Downloading of reference and gene annotation data for pipeline (optional) • TIMING variable

Full box

git pull;

Steps 145 - 150: Steps 145-150: trimming and stepwise mapping of footprint sequence reads

Timing: $\sim 5 \mathrm{~h}$

145. Add Fastq data files to the input_data directory you created (which should also be visible from within the VM). To use the example data set, follow option A. For your own data, use option B.

\section{A. Example data set}

i. Download the example data set using the following commands:

curl 1

http://bioinformatics.erc.monash.edu/TCPseq/vm/input_data.tar | । 


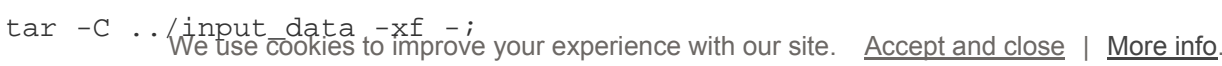

\section{B. Experimental data files}

i. Log back on to the host machine (logout), and copy your Fastq files into the input_data folder you created in Step 143. Files should have the extension . fq.gz and should not be located in subdirectories. Log back on to the VM when finished (vagrant ssh).

Critical step: If you have run a previous analysis on the VM, back up the output if required, and then completely empty the folders input_data, TCPseq/trimmed, TCPseq/mapped and TCPseq/tabulated_data, and delete the file TCPseq/input_filenames_auto.txt before starting a new analysis.

146. Initialize various variables in the current shell session and each time the shell session is restarted.

source TCPseq_config.sh;

Critical step: The TCPseq_config.sh script must be sourced (as above) at the commencement of each new shell session for the TCP-seq pipeline.

\section{Troubleshooting}

147. Trim dubious $3^{\prime}$ basecalls using Trimmomatic and select reads having a poly(A) tail using cutadapt (tail is also trimmed).

cd trimmed;

for $i$ in $\$$ (seq \$FNUM); do I

bn $=\$\left\{F Q \_H N D L[\$ i]\right\} \cdot f q \cdot g z$;

trimmomatic SE -threads $2 \ldots /$ IINPUT_DATADIR/\$\{FQ_FNS[\$i]\} ।

tr_\$bn SLIDINGWINDOW:7:24 MINLEN:26;

cutadapt --discard-untrimmed -a AAAAAAAAAAAA -e $0 . \odot$-0 12 ।

tr_\$bn | gzip > pA_tr_\$bn;

done;

148. Trim away any adaptor sequences from the 5 ' ends of reads, if present (usually $<1 \%$ of reads should exhibit these). Keep only reads whose remaining sequence is $\geq 14 \mathrm{nt}$ long.

cd trimmed;

for $i$ in $\$$ (seq \$FNUM); do I

$\mathrm{bn}=\$\left\{\mathrm{FQ} \_\right.$HNDL $\left.[\$ i]\right\} \cdot \mathrm{fq} \cdot \mathrm{gz}$;

cutadapt -g tCAgAGTTCTACAGTCCGACGATC - e $0 . \odot-011$ pA_tr_\$bn \

| gzip > fpc_pA_tr_\$bn;

trimmomatic SE -threads 2 fpc_pA_tr_\$bn m14_fpc_pA_tr_\$bn V

MINLEN : 14 ;

done;

149. Filter out reads that map to rRNA, tRNA or other structural RNAs, by mapping to the premade yeast index files.

./scripts/stepwise_subtractive_mapping.sh;

Critical step: 


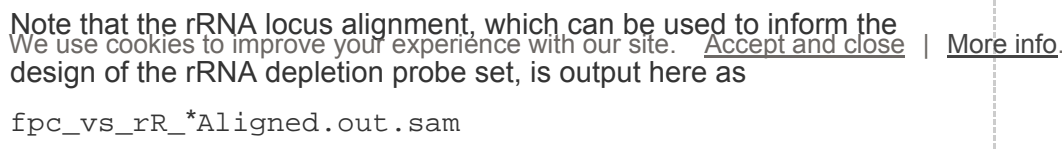

Troubleshooting

150. Take the remaining unmapped reads from Step 149 and align them with the index of spliced transcript sequences with a 1-kb flanking genomic sequence.

cd mapped;

for $i$ in $\$($ seq $\$ F N U M)$; do

$\mathrm{bn}=\$\left\{\mathrm{FQ} \_\right.$HNDL $\left.[\$ i]\right\} ;$

STAR --readFilescommand zcat --genomeDir \$MRI_S --runThreadN I

8 --outFilterMultimapNmax 20000 --readFilesIn noRTS_\$bn.fq.gz \

- - outFileNamePrefix noRTS v orf1000exdub_\$bn 2> ।

noRTS_v_orf1000exdub_\$bn.txt;

done;

Steps 151 - 155: Steps 151-155: processing of the alignments

Timing: $\sim 4 \mathrm{~h}$

151. Run the following Bash code, which executes a Perl script that applies the rules summarized in Supplementary Figure 2 to infer footprint lengths from alignment information.

cd tabulated_data;

for i in \$(seq \$FNUM); do $\backslash$

$\mathrm{bn}=\$\left\{\mathrm{FQ} \_\right.$HNDL $\left.[\$ i]\right\} ;$

cat ...mapped/noRTS_v_orf1000exdub_\$bn\Aligned.out.sam | I

perl ../scripts/sam2table_v2.pl -min 17 -out ।

tbl1_check_\$bn.sam | gzip > tbl1_\$bn\_min17.txt.gz;

done;

152. To obtain the length of the poly $(A)$ tract for alignments ending on $\operatorname{poly}(A)$, run the following Bash code, which executes a Python script that annotates the alignments with genomeencoded downstream poly $(\mathrm{A})$ tract lengths. Output is in zero-based BED format.

cd tabulated_data;

python .../scripts/index_A_tracts.py -p tbl1_-h \$FQ_HNDL_STRNG \

-s_min17.txt.gz -m ।

'../sacCer3/mRNAs_1000flank/mRNAs_1000flank.fa' -f 1000;

153. Run the following Bash code, which executes an R script that collects additional data for the reads, based on transcript-level information. The data set produced is a comprehensive BED file that can be used for various purposes such as calculating read coverage or filtering reads 
flagged at one of the preceding steps (please refer to the information in the table below and see the included readme . txt file for further information on all columns).

cd tabulated_data;

for $i$ in $\$($ seq $\$ F N U M)$; do $\backslash$

$\mathrm{bn}=\$\left\{\mathrm{FQ} \_\mathrm{HNDL}[\$ \mathrm{i}]\right\} ;$

Rscript ../scripts/Process_bed_for_plotting.R I

tbl2_\$bn.bed.gz ।

../sacCer3/annotation/genetable_dist_to_nextgene.txt ।

tbl3_\$bn.bed.gz 1000;

done ;

154. To obtain a transcript-level table of metrics as given in the TCP-seq data browser, it is necessary to specify which samples are derived from SSU, complete ribosome or total translated RNA fractions. Using a text editor or Microsoft Excel, open the file 'input_filenames_auto.txt' (which should have been automatically generated), and fill in the third and fourth columns, in which should be entered the parent sample and fraction type, respectively. Save as a new tab-delimited file in the same folder.

Critical step: Do not save this file under the original name, as that file could be erased and regenerated. An example of a completed table,

'input_filenames_manual.txt', is given along with the example data set.

155. To generate gene-level metrics from the TCP-seq data (1 file per sample), run the following Bash code, which executes an R script for gene-level summarization. Specify the new file name you made in Step 154 as the first argument (i.e., replace 'sample_file. txt' with the file-name), and the desired output file-name prefix as the second argument. Reads can also be prefiltered by any logical ('True'/'False') column in the input BED table; see the included readme file for further instructions.

cd tabulated_data;

python ../scripts/gene_level_metrics.py sample_file.txt ।

glm_out_;

\section{Troubleshooting}

Troubleshooting advice can be found in Table 1.

Table 1: Troubleshooting table.

\section{Timing}

Steps 1-20, fixation of cells: $\sim 5 \mathrm{~h}$

Steps 21-25, lysis of cells: $\sim 2 \mathrm{~h}$

Steps 26-50, isolation of translated mRNA: $\sim 1 \mathrm{~d}$

Steps 51-62, cleavage of translated mRNA with RNase and isolation of SSU and complete

ribosome fractions: $\sim 1 \mathrm{~d}$

Steps $63-72$, isolation of fragmented RNA including SSU and complete ribosome RNA footprints:

$\sim 6 \mathrm{~h}$ 
Steps 73-86, end-repair and polyadenylation of the RNA fragments: $\sim 5 \mathrm{~h}$. Steps $87-103$, synthesis of cDNA and removal of primer excess and RNA: $\sim 3 \mathrm{~h}$

Steps 104-108, circularization of the cDNA: $\sim 7 \mathrm{~h}$

Steps 109-120, rRNA depletion with PDD: $\sim 1 \mathrm{~d}$

Steps 121 and 122, amplification of the cDNA: $\sim 4 \mathrm{~h}$

Steps 123-139, size selection of the amplified cDNA: $\sim 1 \mathrm{~d}$

Steps 140 and 141, high-throughput sequencing: $\sim 3 d$

Steps $142-144$, setting up of the virtual machine for data analysis: $\sim 4 \mathrm{~h}$

Steps $145-150$, trimming and stepwise mapping of footprint sequence reads: $\sim 5 \mathrm{~h}$

Steps 151-155, processing the alignments: $\sim 4 \mathrm{~h}$

Box 1, preparation of sucrose density gradients: $\sim 4 \mathrm{~h}$

Box 2, DNA or RNA cleanup using ethanol precipitation: $\sim 4 \mathrm{~h}$

Box 3, gel filtration of small volumes of DNA and RNA solutions: $\sim 15 \mathrm{~min}$

Box 4, purification of DNA using AMPure XP beads: $\sim 1 \mathrm{~h}$

Box 5 , downloading of reference and gene annotation data for pipeline (optional): variable

\section{Anticipated results}

The protocol is based strictly on the TCP-seq approach used in our original publication for the study of translation dynamics in live yeast cells ${ }^{45}$, and therefore its application as specified will generate similar data. On the metagene level, it can be expected that SSU footprints of a wide (17- to 75-nt) length range will map primarily to mRNA 5' UTRs and associate with scanning SSUs. At start codons, SSU footprints should deconvolute into three major subpopulations of 19, 29 and $37 \mathrm{nt}$, primarily due to variability in 3 ' footprint end protection; these probably derive from different start codon recognition intermediates. Over ORFs and at stop codons, footprints from the SSU fraction can be expected to fall into a major (29-, 30-nt) and a minor (19-, 21-nt) length class; these probably originate from partial elongating ribosome disassembly within ORFs and ribosome recycling at stop codons, respectively. Full ribosomes within ORFs can be expected to generate major long (31-nt) and minor short (21-nt) footprints with 3-nt periodicity over ORFs, comparable to ribosome profiling data ${ }^{85}$. Slightly different footprint lengths of the complete ribosomes can be expected at start and stop codons (21 and $32 \mathrm{nt}$ versus 22 and $31 \mathrm{nt}$, respectively), reflecting differences between initiating and terminating full ribosome-mRNA complexes (Fig. 8a).

The example of S. cerevisiae PAB1 mRNA illustrates TCP-seq data characteristics for individual transcripts with sufficient coverage (Fig. 8a). Shown are read alignments for the SSU and complete ribosome footprint libraries, as well the total translated RNA reference library, based on published data $^{45}$. SSU footprints accumulate mainly in the 5' UTR and at start codons, whereas full ribosome footprints predominate within the ORF, and reference reads cover the entire mRNA lengths relatively evenly. The capacity of the TCP-seq to identify scanning regulatory elements in $5^{\prime}$ UTRs is also evident. PAB1 mRNA features an unusually high density of SSU footprints in its 5' UTR (Fig. 8a), indicative of a possible autoregulatory mechanism involving Pab1 binding to an element in the $5^{\prime}$ UTR of its mRNA, thus probably blocking the progression of scanning complexes ${ }^{45}$. Detailed analyses of SSU footprint characteristics and their density distribution along individual mRNA 5' UTRs can thus help to identify and characterize cases of translational control. A ranking of mRNAs by their ratios of 5' UTR to start codon peak SSU footprint density (Fig. 4b) can assist in shortlisting candidates for such scrutiny.

If the protocol is followed closely, for each 1,000 raw Illumina HiSeq 2500 output reads $~ 9$ (SSU library), 13 (complete ribosome library) and 50 (total translated RNA library) quality-filtered, mRNAmapped reads will be obtained. Because TCP-seq incorporates multiple stages with sample splitting for backup, variable sample recovery during phenol-chloroform extractions, gel filtration and other steps, including variable cycle cDNA amplification, it is not possible to relate sequencing read counts to the absolute amount of the starting material. However, analyzing full ribosome footprints in the ORFs as evenly covered regions, we can apply maximum thresholds for per-gene 
footprint density (i.e., number of footprint $5^{\prime}$ ends per nt of ORF length) and quantify the number of duplicated positions that are obtained with each threshold. Using this approach to estimate library complexity, we find that as the coverage decreases and approaches a minimum, the rate of read duplication stabilizes at $\sim 4 \%$ (Fig. 8 b). Thus, the complete ribosome library is maximally $4 \%$ duplicated and could be sequenced (or amplified) more deeply before approaching saturation. SSU and total translated RNA libraries are expected to have similar duplication rates, as they have a comparable proportion of mRNA reads and are treated identically. The major obstacle to further reduction of the amount of starting material is the detection limits of the SSU peak on a sedimentogram (Fig. 5b). However, if TCP-seq is used to analyze only complete ribosome fraction, the amount of starting material could be downscaled further (at least tenfold) without compromising the library complexity.

\section{References}

1. Gebauer, F., Preiss, T. \& Hentze, M.W. From cis-regulatory elements to complex RNPs and back. Cold Spring Harb. Perspect. Biol. 4, a012245 (2012).

2. Castello, A. et al. Insights into RNA biology from an atlas of mammalian mRNA-binding proteins. Cell 149, 1393-1406 (2012).

3. Hinnebusch, A.G. Translational control 1995-2015: unveiling molecular underpinnings and roles in human biology. RNA 21, 636-639 (2015).

4. Bhat, M. et al. Targeting the translation machinery in cancer. Nat. Rev. Drug Discov. 14, 261 $-278(2015)$

5. Gao, B. \& Roux, P.P. Translational control by oncogenic signaling pathways. Biochim. Biophys. Acta 1849, 753-765 (2015).

6. Proud, C.G. Mnks, elF4E phosphorylation and cancer. Biochim. Biophys. Acta 1849, 766 $-773(2015)$

7. Siddiqui, N. \& Sonenberg, N. Signalling to elF4E in cancer. Biochem. Soc. Trans. 43, 763 $-772(2015)$

8. Rodnina, M.V. \& Wintermeyer, W. Recent mechanistic insights into eukaryotic ribosomes. Curr. Opin. Cell Biol. 21, 435-443 (2009).

9. Hinnebusch, A.G. The scanning mechanism of eukaryotic translation initiation. Annu. Rev. Biochem. 83, 779-812 (2014).

10. Wilson, D.N. \& Doudna Cate, J.H. The structure and function of the eukaryotic ribosome. Cold Spring Harb. Perspect. Biol. 4, a011536 (2012).

11. Jackson, R.J., Hellen, C.U. \& Pestova, T.V. Termination and post-termination events in eukaryotic translation. Adv. Protein Chem. Struct. Biol. 86, 45-93 (2012).

12. Hershey, J.W., Sonenberg, N. \& Mathews, M.B. Principles of translational control: an overview. Cold Spring Harb. Perspect. Biol. 4, a011528 (2012).

13. Steitz, T.A. A structural understanding of the dynamic ribosome machine. Nat. Rev. Mol. Cell Biol. 9, 242-253 (2008).

14. Merrick, W.C. Eukaryotic protein synthesis: still a mystery. J. Biol. Chem. 285, 21197-21201 (2010).

15. Marintchev, A. Roles of helicases in translation initiation: a mechanistic view. Biochim. Biophys. Acta 1829, 799-809 (2013).

16. Hinnebusch, A.G., Ivanov, I.P. \& Sonenberg, N. Translational control by 5'-untranslated regions of eukaryotic mRNAs. Science 352, 1413-1416 (2016). 
17. Vilela, C. \& McCarthy , J.E. Regulation of fungal gene expression via short open reading frames in the mRNA 5'untranslated region. Mol. Microbiol. 49, 859-867 (2003).

18. Hentze, M.W. \& Kuhn, L.C. Molecular control of vertebrate iron metabolism: mRNA-based regulatory circuits operated by iron, nitric oxide, and oxidative stress. Proc. Natl. Acad. Sci. USA 93, 8175-8182 (1996).

19. Kozak, M. Regulation of translation via mRNA structure in prokaryotes and eukaryotes. Gene 361, 13-37 (2005).

20. Pestova, T.V. \& Kolupaeva, V.G. The roles of individual eukaryotic translation initiation factors in ribosomal scanning and initiation codon selection. Genes Dev. 16, 2906-2922 (2002).

21. Kozak, M. A short leader sequence impairs the fidelity of initiation by eukaryotic ribosomes. Gene Expr. 1, 111-115 (1991).

22. Kozak, M. Leader length and secondary structure modulate mRNA function under conditions of stress. Mol. Cell Biol. 8, 2737-2744 (1988).

23. Lozano, G. \& Martinez-Salas, E. Structural insights into viral IRES-dependent translation mechanisms. Curr. Opin. Virol. 12, 113-120 (2015).

24. Pisarev, A.V., Shirokikh, N.E. \& Hellen, C.U. Translation initiation by factor-independent binding of eukaryotic ribosomes to internal ribosomal entry sites. C. R. Biol. 328, 589-605 (2005).

25. Komar, A.A., Mazumder, B. \& Merrick, W.C. A new framework for understanding IRESmediated translation. Gene 502, 75-86 (2012).

26. Gilbert, W.V., Zhou, K., Butler, T.K. \& Doudna, J.A. Cap-independent translation is required for starvation-induced differentiation in yeast. Science 317, 1224-1227 (2007).

27. Shirokikh, N.E. \& Spirin, A.S. Poly(A) leader of eukaryotic mRNA bypasses the dependence of translation on initiation factors. Proc. Natl. Acad. Sci. USA 105, 10738-10743 (2008).

28. Kozak, M. Point mutations define a sequence flanking the AUG initiator codon that modulates translation by eukaryotic ribosomes. Cell 44, 283-292 (1986).

29. Kozak, M. Point mutations close to the AUG initiator codon affect the efficiency of translation of rat preproinsulin in vivo. Nature 308, 241-246 (1984).

30. Kozak, M. Downstream secondary structure facilitates recognition of initiator codons by eukaryotic ribosomes. Proc. Natl. Acad. Sci. USA 87, 8301-8305 (1990).

31. Meyer, K.D. et al. 5' UTR m(6)A promotes Cap-independent translation. Cell 163, 999-1010 (2015).

32. Lee, A.S., Kranzusch, P.J., Doudna, J.A. \& Cate, J.H. elF3d is an mRNA cap-binding protein that is required for specialized translation initiation. Nature 536, 96-99 (2016).

33. Ingolia, N.T. Ribosome footprint profiling of translation throughout the genome. Cell 165, 22 -33 (2016).

34. Ingolia, N.T., Ghaemmaghami, S., Newman, J.R. \& Weissman, J.S. Genome-wide analysis in vivo of translation with nucleotide resolution using ribosome profiling. Science $324,218-223$ (2009).

35. Brar, G.A. \& Weissman, J.S. Ribosome profiling reveals the what, when, where and how of protein synthesis. Nat. Rev. Mol. Cell Biol. 16, 651-664 (2015).

36. Ingolia, N.T. Ribosome profiling: new views of translation, from single codons to genome scale. Nat. Rev. Genet. 15, 205-213 (2014). 
37. Jackson, R. \& Standart, N. The awesome power of ribosome profiling. RNA 21, 652-654 (2015).

38. McGeachy, A.M. \& Ingolia, N.T. Starting too soon: upstream reading frames repress downstream translation. EMBO J. 35, 699-700 (2016).

39. Andreev, D.E. et al. Translation of $5^{\prime}$ leaders is pervasive in genes resistant to elF2 repression. eLife 4, e03971 (2015).

40. Michel, A.M., Ahern, A.M., Donohue, C.A. \& Baranov, P.V. GWIPS-viz as a tool for exploring ribosome profiling evidence supporting the synthesis of alternative proteoforms. Proteomics 15, 2410-2416 (2015).

41. Gao, X., Wan, J. \& Qian, S.B. Genome-wide profiling of alternative translation initiation sites. Methods Mol. Biol. 1358, 303-316 (2016).

42. Vanderperre, B. et al. Direct detection of alternative open reading frames translation products in human significantly expands the proteome. PLoS One 8, e70698 (2013).

43. Shah, P., Ding, Y., Niemczyk, M., Kudla, G. \& Plotkin, J.B. Rate-limiting steps in yeast protein translation. Cell 153, 1589-1601 (2013).

44. Baranov, P.V. \& Loughran, G. Catch me if you can: trapping scanning ribosomes in their footsteps. Nat. Struct. Mol. Biol. 23, 703-704 (2016).

45. Archer, S.K., Shirokikh, N.E., Beilharz, T.H. \& Preiss, T. Dynamics of ribosome scanning and recycling revealed by translation complex profiling. Nature 535, 570-574 (2016).

46. Ingolia, N.T., Brar, G.A., Rouskin, S., McGeachy, A.M. \& Weissman, J.S. The ribosome profiling strategy for monitoring translation in vivo by deep sequencing of ribosome-protected mRNA fragments. Nat. Protoc. 7, 1534-1550 (2012).

47. Dass, C.M. \& Bayley, S.T. A structural study of rat liver ribosomes. J. Cell Biol. 25, 9-22 (1965)

48. Marcus, L., Ris, H., Halvorson, H.O., Bretthauer, R.K. \& Bock, R.M. Occurrence, isolation, and characterization of polyribosomes in yeast. J. Cell Biol. 34, 505-512 (1967).

49. King, H.A. \& Gerber, A.P. Translatome profiling: methods for genome-scale analysis of mRNA translation. Brief Funct. Genomics 15, 22-31 (2016).

50. Pospisek, M. \& Valasek, L. Polysome profile analysis--yeast. Methods Enzymol. 530, 173 -181 (2013).

51. Masek, T., Valasek, L. \& Pospisek, M. Polysome analysis and RNA purification from sucrose gradients. Methods Mol. Biol. 703, 293-309 (2011).

52. Barondes, S.H. \& Nirenberg, M.W. Fate of a synthetic polynucleotide directing cell-free protein synthesis II. Association with ribosomes. Science 138, 813-817 (1962).

53. Steitz, J.A. Polypeptide chain initiation: nucleotide sequences of the three ribosomal binding sites in bacteriophage R17 RNA. Nature 224, 957-964 (1969).

54. Both, G.W., Furuichi, Y., Muthukrishnan, S. \& Shatkin, A.J. Ribosome binding to reovirus mRNA in protein synthesis requires 5' terminal 7-methylguanosine. Cell 6, 185-195 (1975).

55. Wolin, S.L. \& Walter, P. Ribosome pausing and stacking during translation of a eukaryotic mRNA. EMBO J. 7, 3559-3569 (1988).

56. Kozak, M. \& Shatkin, A.J. Characterization of ribosome-protected fragments from reovirus messenger RNA. J. Biol. Chem. 251, 4259-4266 (1976).

57. Kozak, M. \& Shatkin, A.J. Sequences and properties of two ribosome binding sites from the small size class of reovirus messenger RNA. J. Biol. Chem. 252, 6895-6908 (1977). 
58. Kolupaeva, V.G., Pestova, T.V. \& Hellen, C.U. An enzymatic footprinting analysis of the
. We use cookites to improve your experience with our site. Accept and close interaction of $40 \mathrm{~S}$ ribosomal subunits with the internal ribosomal entry site of hepatitis $C$ virus. J. Virol. 74, 6242-6250 (2000).

59. Karmakar, S. et al. Organocatalytic removal of formaldehyde adducts from RNA and DNA bases. Nat. Chem. 7, 752-758 (2015).

60. Solomon, M.J. \& Varshavsky, A. Formaldehyde-mediated DNA-protein crosslinking: a probe for in vivo chromatin structures. Proc. Natl. Acad. Sci. USA 82, 6470-6474 (1985).

61. Viswanathan, R., Hoffman, E.A., Shetty, S.J., Bekiranov, S. \& Auble, D.T. Analysis of chromatin binding dynamics using the crosslinking kinetics (CLK) method. Methods 70, 97 -107 (2014)

62. Moller, K., Rinke, J., Ross, A., Buddle, G. \& Brimacombe, R. The use of formaldehyde in RNA-protein cross-linking studies with ribosomal subunits from Escherichia coli. Eur. J. Biochem. 76, 175-187 (1977).

63. Sen, N.D., Zhou, F., Ingolia, N.T. \& Hinnebusch, A.G. Genome-wide analysis of translational efficiency reveals distinct but overlapping functions of yeast DEAD-box RNA helicases Ded1 and elF4A. Genome Res. 25, 1196-1205 (2015).

64. Schmiedeberg, L., Skene, P., Deaton, A. \& Bird, A. A temporal threshold for formaldehyde crosslinking and fixation. PLoS One 4, e4636 (2009).

65. Poorey, K. et al. Measuring chromatin interaction dynamics on the second time scale at single-copy genes. Science 342, 369-372 (2013).

66. Evers, D.L., Fowler, C.B., Cunningham, B.R., Mason, J.T. \& O'Leary, T.J. The effect of formaldehyde fixation on RNA: optimization of formaldehyde adduct removal. J. Mol. Diagn. 13, 282-288 (2011).

67. Furey, T.S. ChIP-seq and beyond: new and improved methodologies to detect and characterize protein-DNA interactions. Nat. Rev. Genet. 13, 840-852 (2012).

68. Park, P.J. ChIP-seq: advantages and challenges of a maturing technology. Nat. Rev. Genet. 10, 669-680 (2009).

69. Archer, S.K., Shirokikh, N.E., Hallwirth, C.V., Beilharz, T.H. \& Preiss, T. Probing the closedloop model of mRNA translation in living cells. RNA Biol. 12, 248-254 (2015).

70. Strunk, B.S., Novak, M.N., Young, C.L. \& Karbstein, K. A translation-like cycle is a quality control checkpoint for maturing 40S ribosome subunits. Cell 150, 111-121 (2012).

71. Valasek, L., Szamecz, B., Hinnebusch, A.G. \& Nielsen, K.H. In vivo stabilization of preinitiation complexes by formaldehyde cross-linking. Methods Enzymol. 429, 163-183 (2007).

72. Weber, L.A., Hickey, E.D. \& Baglioni, C. Influence of potassium salt concentration and temperature on inhibition of mRNA translation by 7-methylguanosine5'-monophosphate. $\mathrm{J}$. Biol. Chem. 253, 178-183 (1978).

73. Tulin, E.E., Tsutsumi, K. \& Ejiri, S. Continuously coupled transcription-translation system for the production of rice cytoplasmic aldolase. Biotechnol. Bioeng. 45, 511-516 (1995).

74. Louie, M.K., Francisco, J.S., Verdicchio, M., Klippenstein, S.J. \& Sinha, A. Dimethylamine addition to formaldehyde catalyzed by a single water molecule: a facile route for atmospheric carbinolamine formation and potential promoter of aerosol growth. J. Phys. Chem. A 120, 1358-1368 (2016). 
75. Ugye, J.T., Uzairu, A Idris, S.O \& Kwanashie, H.O. Temperature effects on the rate of reaction of plasma albumin with formaldehyde in water solution and ethanol-water-mixtures.

Chem. J. 03, 128-132 (2013).

76. Archer, S.K., Shirokikh, N.E. \& Preiss, T. Probe-Directed Degradation (PDD) for flexible removal of unwanted cDNA sequences from RNA-Seq libraries. Curr. Protoc. Hum. Genet. 85, Unit 11.15 (2015).

77. Anisimova, V.E., Barsova, E.V., Bogdanova, E.A., Lukyanov, S.A. \& Shcheglov, A.S. Thermolabile duplex-specific nuclease. Biotechnol. Lett. 31, 251-257 (2009).

78. Archer, S.K., Shirokikh, N.E. \& Preiss, T. Selective and flexible depletion of problematic sequences from RNA-seq libraries at the cDNA stage. BMC Genom. 15, 401 (2014).

79. Nagalakshmi, U. et al. The transcriptional landscape of the yeast genome defined by RNA sequencing. Science 320, 1344-1349 (2008).

80. Kertesz, M. et al. Genome-wide measurement of RNA secondary structure in yeast. Nature 467, 103-107 (2010)

81. Dever, T.E., Kinzy, T.G. \& Pavitt, G.D. Mechanism and regulation of protein synthesis in Saccharomyces cerevisiae. Genetics 203, 65-107 (2016).

82. Altmann, M. \& Linder, P. Power of yeast for analysis of eukaryotic translation initiation. J. Biol. Chem. 285, 31907-31912 (2010).

83. Lindqvist, L. \& Pelletier, J. Inhibitors of translation initiation as cancer therapeutics. Future Med. Chem. 1, 1709-1722 (2009).

84. Popa, A., Lebrigand, K., Barbry, P. \& Waldmann, R. Pateamine A-sensitive ribosome profiling reveals the scope of translation in mouse embryonic stem cells. BMC Genom. 17, 52 (2016).

85. Lareau, L.F., Hite, D.H., Hogan, G.J. \& Brown, P.O. Distinct stages of the translation elongation cycle revealed by sequencing ribosome-protected mRNA fragments. eLife $\mathbf{3}$, e01257 (2014).

86. Ingolia, N.T., Lareau, L.F. \& Weissman, J.S. Ribosome profiling of mouse embryonic stem cells reveals the complexity and dynamics of mammalian proteomes. Cell 147, 789-802 (2011).

87. Gao, X. et al. Quantitative profiling of initiating ribosomes in vivo. Nat. Methods 12, 147-153 (2015).

88. Bartholomaus, A., Del Campo, C. \& Ignatova, Z. Mapping the non-standardized biases of ribosome profiling. Biol. Chem. 397, 23-35 (2016).

89. Gerashchenko, M.V. \& Gladyshev, V.N. Translation inhibitors cause abnormalities in ribosome profiling experiments. Nucleic Acids Res. 42, e134 (2014).

90. Weinberg, D.E. et al. Improved ribosome-footprint and mRNA measurements provide insights into dynamics and regulation of yeast translation. Cell Rep. 14, 1787-1799 (2016).

91. Hussmann, J.A., Patchett, S., Johnson, A., Sawyer, S. \& Press, W.H. Understanding biases in ribosome profiling experiments reveals signatures of translation dynamics in yeast. PLoS Genet. 11, e1005732 (2015).

92. Yamamoto, H. et al. Structure of the mammalian $80 \mathrm{~S}$ initiation complex with initiation factor 5B on HCV-IRES RNA. Nat. Struct. Mol. Biol. 21, 721-727 (2014).

93. Weisser, M., Voigts-Hoffmann, F., Rabl, J., Leibundgut, M. \& Ban, N. The crystal structure of the eukaryotic $40 \mathrm{~S}$ ribosomal subunit in complex with elF1 and elF1A. Nat. Struct. Mol. Biol. 20, 1015-1017 (2013). 
94. Muhs, M. et al. Cryo-EM of ribosomal 80S complexes with termination factors reveals the translocated cricket paralysis virus IRES. Mol. Cell 57, 422-432 (2015).

95. des Georges, A. et al. Structure of the mammalian ribosomal pre-termination complex associated with eRF1.eRF3.GDPNP. Nucleic Acids Res. 42, 3409-3418 (2014).

96. Lomakin, I.B. \& Steitz, T.A. The initiation of mammalian protein synthesis and mRNA scanning mechanism. Nature 500, 307-311 (2013).

97. Koutmou, K.S. et al. Ribosomes slide on lysine-encoding homopolymeric A stretches. eLife 4, e05534 (2015).

98. Parenteau, J. et al. Deletion of many yeast introns reveals a minority of genes that require splicing for function. Mol. Biol. Cell 19, 1932-1941 (2008).

99. Kanitz, A. et al. Comparative assessment of methods for the computational inference of transcript isoform abundance from RNA-seq data. Genome Biol. 16, 150 (2015).

100. Martinez-Nunez, R.T. \& Sanford, J.R. Studying isoform-specific mRNA recruitment to polyribosomes with Frac-seq. Methods Mol. Biol. 1358, 99-108 (2016).

101. Wang, H., McManus, J. \& Kingsford, C. Isoform-level ribosome occupancy estimation guided by transcript abundance with Ribomap. Bioinformatics 32, 1880-1882 (2016).

102. Spealman, P., Wang, H., May, G., Kingsford, C. \& McManus, C.J. Exploring ribosome positioning on translating transcripts with ribosome profiling. Methods Mol. Biol. 1358, 71-97 (2016).

103. Ji, Z., Song, R., Huang, H., Regev, A. \& Struhl, K. Transcriptome-scale RNase-footprinting of RNA-protein complexes. Nat. Biotechnol. 34, 410-413 (2016).

104. Berthelot, K., Muldoon, M., Rajkowitsch, L., Hughes, J. \& McCarthy, J.E.G. Dynamics and processivity of $40 \mathrm{~S}$ ribosome scanning on mRNA in yeast. Mol. Microbiol. 51, 987-1001 (2004).

105. Karpinets, T.V., Greenwood, D.J., Sams, C.E. \& Ammons, J.T. RNA:protein ratio of the unicellular organism as a characteristic of phosphorous and nitrogen stoichiometry and of the cellular requirement of ribosomes for protein synthesis. BMC Biol. 4, 30 (2006).

106. Xiao, Z., Zou, Q., Liu, Y. \& Yang, X. Genome-wide assessment of differential translations with ribosome profiling data. Nat. Commun. 7, 11194 (2016).

107. Young, D.J., Guydosh, N.R., Zhang, F., Hinnebusch, A.G. \& Green, R. Rli1/ABCE1 recycles terminating ribosomes and controls translation reinitiation in 3'UTRs in vivo. Cell 162, 872 $-884(2015)$

108. Collins, K. \& Nilsen, T.W. Enzyme engineering through evolution: thermostable recombinant group II intron reverse transcriptases provide new tools for RNA research and biotechnology. RNA 19, 1017-1018 (2013).

109. Mohr, S. et al. Thermostable group II intron reverse transcriptase fusion proteins and their use in cDNA synthesis and next-generation RNA sequencing. RNA 19, 958-970 (2013).

110. Nottingham, R.M. et al. RNA-seq of human reference RNA samples using a thermostable group II intron reverse transcriptase. RNA 22, 597-613 (2016).

111. Qin, Y. et al. High-throughput sequencing of human plasma RNA by using thermostable group II intron reverse transcriptases. RNA 22, 111-128 (2016).

112. Chung, B.Y. et al. The use of duplex-specific nuclease in ribosome profiling and a userfriendly software package for Ribo-seq data analysis. RNA 21, 1731-1745 (2015). 
113. Yi, H. et al. Duplex-specific nuclease efficiently removes rRNA for prokaryotic RNA-seq. Nucleic Acids Res. 39, e140 (2011).

114. Bogdanova, E.A., Shagin, D.A. \& Lukyanov, S.A. Normalization of full-length enriched cDNA. Mol. Biosyst. 4, 205-212 (2008).

115. Zhulidov, P.A. et al. Simple cDNA normalization using kamchatka crab duplex-specific nuclease. Nucleic Acids Res. 32, e37 (2004).

116. Zhang, Z., Hesselberth, J.R. \& Fields, S. Genome-wide identification of spliced introns using a tiling microarray. Genome Res. 17, 503-509 (2007).

Download references

\section{Acknowledgments}

This work was supported by an ARC Discovery Grant (DP1300101928) and an NHMRC Senior Research Fellowship (514904) awarded to T.P. N.E.S. was supported by a Go8 European Fellowship. We acknowledge technical support from the Australian Cancer Research Foundation Biomolecular Resource Facility (John Curtin School of Medical Research, Australian National University) and S. Androulakis at the Monash Bioinformatics Platform.

\section{Author information}

These authors contributed equally to this work.

Nikolay E Shirokikh \& Stuart K Archer

\section{Affiliations}

EMBL-Australia Collaborating Group, Department of Genome Sciences, The John Curtin School of Medical Research, The Australian National University, Canberra, Australian Capital Territory, Australia.

Nikolay E Shirokikh \& Thomas Preiss

State Socio-Humanitarian University, Kolomna, Russia.

Nikolay E Shirokikh

Monash Bioinformatics Platform, Monash University, Melbourne, Victoria, Australia.

Stuart K Archer \& David Powell

Development and Stem Cells Program, Monash Biomedicine Discovery Institute and Department of Biochemistry and Molecular Biology, Monash University, Melbourne, Victoria, Australia.

Traude H Beilharz

Victor Chang Cardiac Research Institute, Darlinghurst, New South Wales, Australia. Thomas Preiss

\section{Contributions}

N.E.S., S.K.A. and T.P. developed the protocol; N.E.S. and S.K.A. performed the biochemical experiments; S.K.A. and D.P. developed the bioinformatics analysis framework and analyzed the data; and N.E.S., S.K.A., T.H.B. and T.P. discussed and interpreted results. All authors contributed to the writing of the manuscript.

\section{Competing financial interests}

The authors declare no competing financial interests. 


\section{Corresponding author \\ Accept and close | More info} Correspondence to: Thomas Preiss

\section{Supplementary information}

\section{Supplementary Figures}

1. Supplementary Figure 1: Library construction approach used in TCP-seq. (150 KB) Overview of the library construction steps used in TCP-seq, starting from the de-blocked RNA fragments isolated from the SSU, complete ribosome or total translated RNA fractions (Step 73), and ending with the amplified, size-selected strand-specific library (Step 128).

2. Supplementary Figure 2: Schematic of algorithm for inferring footprint lengths from alignments. (320 KB)

Representative alignments of reads (top sequences) to a reference (bottom sequences) are given and the nucleotides or gaps counted towards the footprint lengths is indicated in red. To the right of each alignment is its corresponding CIGAR representation, output by the aligner. $3^{\prime}$ poly $(A)$ tracts that were mismatched with the reference (but not trimmed away in earlier steps due to an erroneous internal $\mathrm{G}$ or $\mathrm{T}$ base-call) were trimmed in this step also.

\section{PDF files}

1. Supplementary Figures and Tables (545 KB)

Supplementary Figures 1 and 2, and Supplementary Table 1.

Nature Protocols $\quad$ ISSN 1754-2189 EISSN 1750-2799

\section{SPRINGER NATURE}

(C) 2017 Macmillan Publishers Limited, part of Springer Nature. All rights reserved. partner of AGORA, HINARI, OARE, INASP, ORCID, CrossRef, COUNTER and COPE 